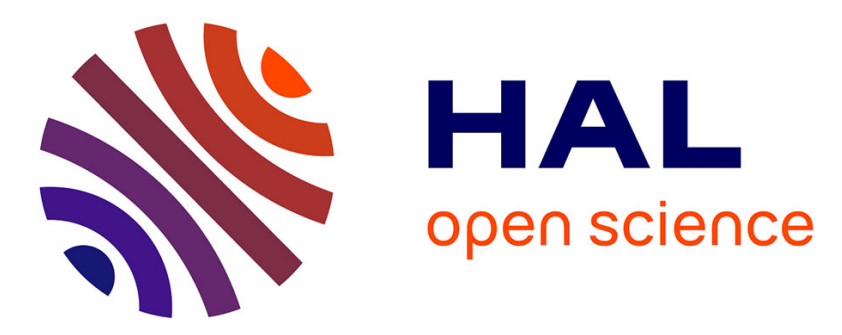

\title{
Effect of spray cooling on heat transfer in a two-phase helium flow
}

\author{
Sylvain Perraud, Laurent Puech, Pierre Thibault, Bernard Rousset,
} Pierre-Etienne Wolf

\section{- To cite this version:}

Sylvain Perraud, Laurent Puech, Pierre Thibault, Bernard Rousset, Pierre-Etienne Wolf. Effect of spray cooling on heat transfer in a two-phase helium flow. Cryogenics, 2013, 57, pp.74-87. 10.1016/j.cryogenics.2013.05.006 . hal-00723000

\section{HAL Id: hal-00723000 https://hal.science/hal-00723000}

Submitted on 6 Aug 2012

HAL is a multi-disciplinary open access archive for the deposit and dissemination of scientific research documents, whether they are published or not. The documents may come from teaching and research institutions in France or abroad, or from public or private research centers.
L'archive ouverte pluridisciplinaire HAL, est destinée au dépôt et à la diffusion de documents scientifiques de niveau recherche, publiés ou non, émanant des établissements d'enseignement et de recherche français ou étrangers, des laboratoires publics ou privés. 


\title{
Droplets mediated thermal transfer from an atomized diphasic helium flow
}

\author{
S. Perraud ${ }^{\mathrm{a}, \mathrm{b}}$, L. Puech ${ }^{\mathrm{a}, 1}$, P. Thibault ${ }^{\mathrm{a}, \mathrm{b}, 2}$, B. Rousset ${ }^{\mathrm{b}}$, P.E. Wolf ${ }^{\mathrm{a}, *}$ \\ ${ }^{a}$ Institut Néel, CNRS et Univ. Grenoble 1, BP166, F-38042 Grenoble Cedex 9, France \\ ${ }^{b}$ CEA Grenoble-UMRE 9006, INAC, Service des Basses Températures, 17 rue des \\ Martyrs, 38054 Grenoble-Cedex 9, France
}

\begin{abstract}
In this paper, we describe an experimental study of the phenomenon of spray cooling in the case of liquid helium, either normal or superfluid, and its relationship to the heat transfer between an atomized diphasic flow contained in a long pipe, and the pipe walls. This situation is discussed in the context of the cooling of the superconducting magnets of the Large Hadron Collider (LHC). Experiments were conducted in a test loop reproducing the LHC cooling system, in which the vapor velocity and temperature could be varied in a large range. Shear induced atomization results in the generation of a droplet mist which was characterized by optical means. The thin liquid film deposited on the walls by the mist was measured using interdigitated capacitors. The cooling power of the mist was measured using thermal probes, and correlated to the local mist density. Analysis of the results shows that superfluidity has only a limited influence on both the film thickness and the mist cooling power. Using a simple model, we show that the phenomenon of spray cooling accounts for the measured non linearity of the global heat transfer. Finally, we discuss the relevance of our results for cooling the final focus magnets (inner triplets) in an upgraded version of the LHC.
\end{abstract}

Keywords: Spray cooling, Atomization, Multiphase flow, Superfluid heat transport, Film flow

${ }^{*}$ Tel: 33476881273 ; fax 3345387087

email: pierre-etienne.wolf@grenoble.cnrs.fr

${ }^{1}$ deceased, December $2^{\text {nd }}, 2008$

${ }^{2}$ present address: Univ. Grenoble 1, LIPhy UMR 5588, Grenoble, F-38041, France 


\section{Introduction}

Many large scale cryogenic facilities use vaporization of circulating liquid helium to cool cryogenic devices. This is in particular the case of the Large Hadron Collider (LHC) at CERN, the magnets of which are cooled by a pipe containing a two-phase (vapor-superfluid) stratified flow of ${ }^{4} \mathrm{He}$ pumped down to $1.8 \mathrm{~K}$. This paper reports on experiments carried out in a similar geometry, in the situation where the vapor velocity is large enough to partly atomize the liquid flow into a mist of small droplets. Our aim is to investigate how the deposition of these droplets on the pipe walls improves the heat transfer between these walls and the ${ }^{4} \mathrm{He}$ flow. This question might be relevant for future developments of the LHC, where, in contrast to the present situation, the vapor velocity could exceed the atomization threshold.

The problem involves two complex phenomena, spray generation by atomization and spray cooling. For usual fluids, atomization of a stratified flow of liquid (e.g. water) by a gas (e.g. air) has been extensively studied due to its numerous practical applications. This also holds true for spray cooling, where heat is efficiently removed from a hot wall by vaporization of impinging liquid droplets, with many applications from cooling of hot metal in steel industries to efficient cooling of power electronic circuits [1]. However, it is difficult, if not impossible, to extend the results of such studies to the case of liquid helium. The reason is the very low surface tension of helium, as compared to other fluids, which renders an extrapolation hazardous, as the quantitative influence of this parameter on atomization remains a subject of debate. Furthermore, the superfluidity of helium may affect both the atomization process and the spreading of droplets on the walls. Therefore, the influence of atomization on heat transfer in a diphasic superfluid helium flow has to be investigated experimentally.

The primary goal of our experiment was thus to measure the thermal exchange in a realistic situation, close to the actual LHC cooling scheme, integrating both the atomization process and the spray deposition due to the transport of droplets by the turbulent vapor. As a consequence, this experiment differs from dedicated spray cooling studies on usual fluids, where the incident mass flux is generated by a controlled source and directly impinges the wall. Despite this limitation, direct comparison of the behavior for normal and superfluid liquid in similar atomization conditions shows that superfluidity has only a weak influence on the global properties measured. This suggests that the droplets interaction with the walls is not strongly affected 
by the nature of the liquid, either superfluid or normal. This conclusion is of interest for possible uses of spray cooling by superfluid helium, for example in spatial (gravityless) applications, where spray cooling has, up to now, only been used with classical cryogenic liquids (e.g. hydrogen [2]).

This paper is organized as follows. We first summarize the initial motivations for our work. We then give the necessary background by discussing on theoretical grounds the different steps involved in the deposition of a liquid film due to atomization, and its expected influence on the thermal transfer. We then describe the cryogenic system, focusing on its capabilities in terms of atomization, and the experimental tools used to probe the relationship between atomization and thermal transfer, namely the optical set-up for the characterization of the droplets mist, the surface capacitances for detecting the film formation, and the thermal probes for measuring the heat transfer. In the results section, we first focus on the spray generation and its dependence on the physical control parameters. We then discuss how the cooling power of the mist and the thickness of the film depend on the degree of atomization, and on the nature of the liquid. Next, in the superfluid phase, we use a simple model for heat transfer to compare the locally measured cooling power of the mist to the directly measured global heat transfer between the pipe and the diphasic flow. Finally, we discuss the relevance of our results for cooling the final focus magnets (inner triplets) in an upgraded version of the LHC.

\section{Motivations}

Our study is connected to the cooling of superconducting magnets of the Large Hadron Collider (LHC) at CERN [3, 4]. The LHC magnets are immersed in pressurized superfluid ${ }^{4} \mathrm{He}$, which is cooled by a $54 \mathrm{~mm}$ diameter pipe containing a two-phase (vapor-superfluid) flow of ${ }^{4} \mathrm{He}$ pumped down to 1.8 K. Along the LHC ring, liquid helium is injected into the pipe approximately every $100 \mathrm{~m}$ and helium vapor is pumped $100 \mathrm{~m}$ away. The heat dissipated by the magnets is absorbed by the vaporization of the flowing saturated superfluid liquid, resulting in a diphasic flow of liquid and vapor along the pipe. A key factor limiting the magnets temperature is the thermal exchange between the magnets bath and the flowing liquid. In normal operating conditions, the heat load is of order $0.5 \mathrm{~W} / \mathrm{m}$, corresponding to a total heat load of $50 \mathrm{~W}$ along one pipe segment. The resulting vapor flow corresponds to a maximal vapor velocity $V$ smaller than $2 \mathrm{~m} / \mathrm{s}$. In these con- 
ditions, the diphasic flow is stratified, the liquid flowing in the bottom part of the pipe and the vapor above. The heat exchange is then limited by the surface wetted by the liquid (typically 10-20\% of the pipe total surface). For the nominal heat load of $0.5 \mathrm{~W} / \mathrm{m}$, this results in a temperature difference between the diphasic flow and the magnet bath of less than $0.1 \mathrm{~K}$, consistent with the magnet operation temperature $(1.9 \mathrm{~K})$. However, thermal exchange is expected to become an issue in an upgraded version of LHC, where the linear heat load for the final focus magnets is expected to range between 10 and $100 \mathrm{~W} / \mathrm{m}$ [5].

Previous experiments conducted in a test pipe built at CEA-Grenoble have shown that, for vapor velocities larger than $\simeq 5 \mathrm{~m} / \mathrm{s}$, the global heat transfer between a pressurized helium bath and the pipe is larger than predicted by the stratified model [6]. Following experiments combining global thermal measurements and optical observations [7] have shown that this improvement results from the atomization of liquid droplets from the bulk liquid by the fast vapor stream, and their deposition onto the walls. In an ideal case, all the pipe perimeter could be wetted due to such a process, increasing the wetted surface by a large factor. This could suggest that atomization might improve thermal exchange up to the level required by the planned upgrade.

However, this improvement cannot hold up to arbitrary large heat fluxes. Beyond some threshold, which depends on the droplets mist density, all the incident droplets will be directly evaporated, and no liquid film will form. The walls temperature is thus expected to vary non linearly with the heat applied, in qualitative agreement with our earlier observations $[6,8]$. This phenomenon limits the ability of atomization to improve thermal exchange, but in a way which, as discussed in $\S 3$, would be extremely difficult to predict theoretically.

This has motivated the experiment described in this paper, which consists in an extensive quantitative study of the contribution of droplets to the heat transfer. The measurements, which were performed during a single one-month long run, rely on a new cryogenic facility extending the available range of physical parameters controlling atomization, such as the vapor velocity and the temperature. In particular, unlike in the previous experiments $[6,7]$, the regime of atomization could be reached even in the normal fluid region of the phase diagram. Another improvement is the use of new probes measuring locally the thermal transfer between a heated surface and the flow, as well as the thickness of the film deposited on an unheated sur- 
face [9]. By correlating these measurements to the optical characterization of the droplets mist facing the probes, we can evaluate the efficiency of the deposition process in terms of film formation and cooling power, and assay its sensitivity to the superfluidity of the liquid. In a last step, we combine this knowledge with the optically measured mist stratification across the pipe height to predict the dependence of the global heat exchange on the applied heat flux, and compare this prediction to our observations.

\section{Theoretical background}

In this section, we describe the two steps involved in the improvement of heat transfer due to droplets: production of droplets from the liquid free surface by atomization and turbulent transport of these droplets within the pipe, then spray cooling of the pipe walls by these droplets.

Blowing a fast enough gas stream parallel to the free surface of a liquid results in the atomization of liquid into a mist of droplets. This takes place through two successive instabilities as the gas velocity is increased. First, waves appear on the surface above a velocity threshold, due to a KelvinHelmoltz like instability [10]. Above a second threshold, the waves are themselves destabilized leading to the formation of ligaments, which are stretched and sheared into droplets by the gas stream. The spatial distribution of droplets above the liquid involves complex mechanisms, such as the diffusion of droplets due to the gas turbulence, their settling due to gravity, or their deposition on walls. In the simplest approach, where the deposition is negligible and droplets mostly fall back on the liquid, the competition between diffusion and gravity results in an exponential stratification of the mist over a characteristic height which depends on the droplets size [11]. However, coalescence between droplets and their interaction with the turbulent structures might modify this simple picture. Therefore, direct measurements are necessary to characterize the distribution of droplets throughout the pipe cross-section.

In a second step, the droplets mist acts as a source for spray cooling the pipe walls. Spray cooling, the process by which a heated surface exposed to a flow of droplets is cooled by vaporization of these droplets, is characterized by the so-called spray cooling curve, which measures the excess wall temperature with respect to the spray temperature as a function of the applied heat flux. A fundamental quantity describing this curve is the critical heat flux 
(CHF). Below the CHF, a liquid film exists on the surface, and all the heat is evacuated by vaporization. Above the CHF, the surface is dry and heat is at least partly evacuated by thermal transfer to the vapor. For usual liquids, the spray cooling curve depends on many complex phenomena [1]. Below the $\mathrm{CHF}$, the excess wall temperature varies non linearly with the heat flux reflecting different heat transfer mechanisms within the liquid film, conduction or convection for low fluxes or two-phases processes, such as boiling, for larger ones. The $\mathrm{CHF}$ itself depends on which fraction of the incident liquid flux is finally available for vaporization, i.e. on the probability that droplets bounce on the surface or splash emitting secondary droplets which are lost for the cooling process. Studies on individual droplets $[12,13,14]$ have shown that these different processes depend on the surface state (hot or cold, dry or wet, covered by a thin or a thick film). In a real spray, they could be further modified due to interactions between droplets [15]. As a result, the spray efficiency, defined as the ratio of the CHF to $L \Phi_{i n c}$, the product of the massic latent heat to the incident liquid mass flow, is a quantity which is measured by dedicated studies rather than theoretically predicted.

In our experimental situation, unlike in such dedicated studies, the liquid flux $\Phi_{i n c}$ impinging on the pipe walls is not controlled, but has to be computed from the liquid volume fraction transported by the droplets and their average impact velocity $v_{i m p}$ due to turbulent deposition. For droplets small enough to follow the gas isotropic turbulence, $v_{i m p}$ is expected to be of order the fluctuation of the axial vapor velocity, $\delta v_{\text {r.m.s. }}=\left(\delta v_{z}^{2}\right)^{1 / 2}[16,17]$, but could be reduced due to inertia for large ones [18].

Helium has specific properties which make it difficult to extrapolate the results obtained with other fluids. Concerning the evaluation of the incident liquid flux, the low surface tension of helium, as compared to e.g., water, combined with the unsettled functional dependence of the droplets size on this physical parameter $[10,19]$, does not allow to predict the droplets volume fraction from experiments on water-air diphasic flows [20]. Furthermore, for droplets of given size, the low viscosity of the vapor as compared to air makes inertia effects potentially more important. Concerning the spray efficiency, the low viscosity and surface tension of helium may give rise to a specific spreading or splashing behaviour. Even larger modifications could occur in the superfluid phase. Indeed, at zero temperature, when no viscous dissipation is possible, it has been theoretically shown that a superfluid droplet should spread much faster than a viscous droplet [21].

In this paper, we use optical means to directly characterize the droplets 


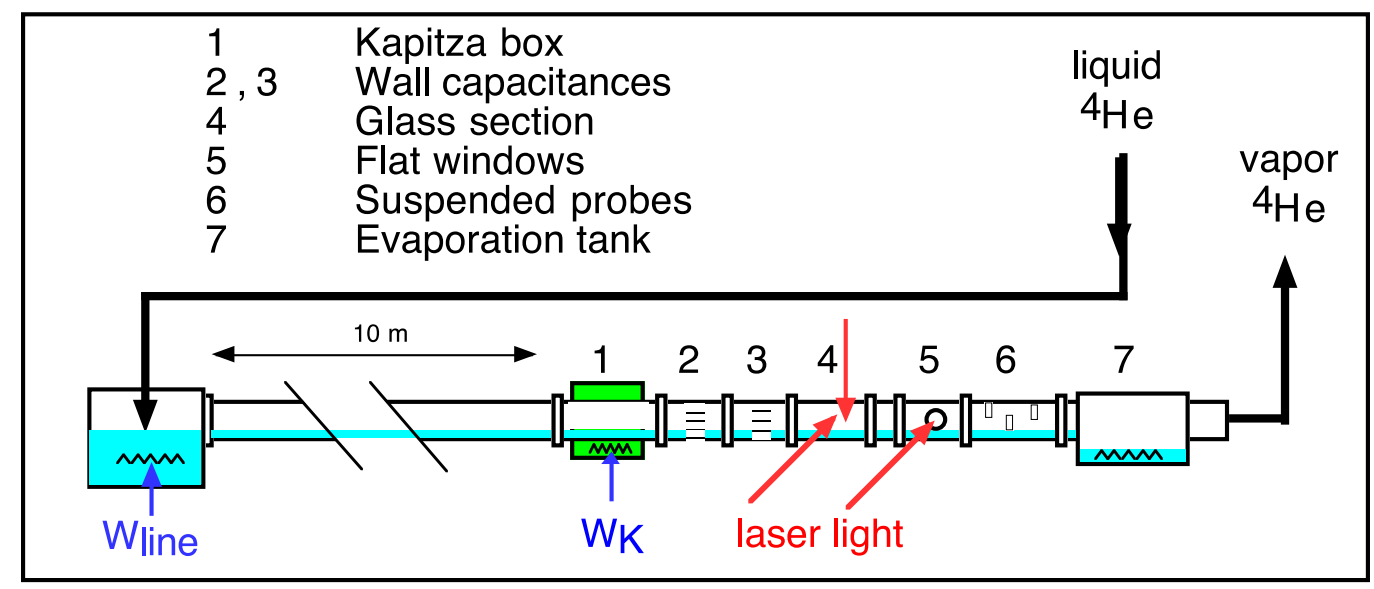

Figure 1: Schematics of the Cryoloop pipe. The diphasic helium flow is produced by partial vaporization of the injected liquid. The properties of the atomized mist are measured $10 \mathrm{~m}$ downstream. The global heat transfer is measured by the Kapitza box (1). The liquid film deposited from the mist is measured in sections (2), (3), and (6) by interdigitated capacitive gauges. The local contribution of the mist to the heat transfer is measured in section (6) by suspended thermal probes. The mist is optically characterized in sections (4) and (5).

spatial distribution within the pipe, as well as their axial velocity and its fluctuations, bypassing the need for any extrapolation of data on usual fluids. We can thus compare in the normal and superfluid phases the film thickness for an unheated surface, and the critical heat flux for a heated surface, for similar spray conditions. This comparison suggests that superfluidity has little influence, if any, on the splashing or spreading processes.

\section{Experimental}

\subsection{Flow generation}

Our experiments were performed in the Cryoloop test line installed at the Service des Basses Températures at CEA-Grenoble. The $40 \mathrm{~mm}$ diameter pipe containing the diphasic helium was installed in a $10 \mathrm{~m}$ long quasi-horizontal cryostat ${ }^{3}$ (figure 1).

\footnotetext{
${ }^{3}$ A slight downwards slope (-0.6\%) ensures a gravitationally driven flow at low vapor velocity.
} 
A specially developed refrigerator [22] with large cooling power $(400 \mathrm{~W}$ at $1.8 \mathrm{~K}$ ) delivers a controllable flow of liquid of up to approximately $20 \mathrm{~g} / \mathrm{s}$ to one end of the pipe. There, this liquid is partly evaporated by a heater in order to obtain downstream an essentially stratified diphasic flow. At the other end of the pipe, the remaining liquid is evaporated, and the exiting vapor is pumped through cold compressors followed by a room temperature pump. This sets the pressure, hence the temperature, inside the pipe.

The physical parameters controlling atomization are the vapor velocity, the saturated vapor density, and the level of liquid. The control parameters are the power $W_{\text {line }}$ applied at the pipe inlet, the injected liquid mass flow $\dot{m}$, and the temperature $T$ of the diphasic helium, which sets the vapor density $\rho_{\mathrm{v}}(T)$. The two first parameters determine the mass flow rates of vapor ${ }^{4}$ $\left(\dot{m}_{\mathrm{v}}=W_{\text {line }} / L\right)$, and of liquid $\left(\dot{m}_{l}=\dot{m}-\dot{m}_{\mathrm{v}}\right)$ inside the pipe. The average vapor velocity is then $V_{\mathrm{v}} \approx \dot{m}_{\mathrm{v}} /\left(\rho_{\mathrm{v}}(T) . S\right)$, where $S$ is the pipe cross-section (only a small fraction of it being occupied by the liquid phase). Finally, for given vapor velocity and temperature, the liquid level is varied by changing the liquid mass flow $\dot{m}_{l}$.

At a given temperature, the vapor velocity is proportional to $W_{\text {line }}$, up to the complete vaporization of the injected liquid $\left(W_{\text {line }}=\dot{m} / L \approx 400 \mathrm{~W}\right.$ for $\dot{m}=18 \mathrm{~g} / \mathrm{s}$ ). At $1.8 \mathrm{~K}$, the corresponding maximal vapor velocity is about $30 \mathrm{~m} / \mathrm{s}$. Note that this is more than 15 times the vapor velocity at LHC ( $W_{\text {line }} \leq 50 \mathrm{~W}$, pipe section $70 \%$ larger than for Cryoloop).

Because the vapor density increases from 0.45 to $3.4 \mathrm{~g} / \mathrm{cm}^{3}$ between 1.8 and $2.8 \mathrm{~K}$, this maximal velocity decreases with increasing temperature. In practice, atomization requires a minimal liquid level, reducing the operational range of velocities. Typically, for a liquid height of $3.5 \mathrm{~mm}$ (corresponding to a wetted fraction of the pipe of $20 \%$ ), the maximal velocity decreases from about $18 \mathrm{~m} / \mathrm{s}$ at $1.8 \mathrm{~K}$ to $4 \mathrm{~m} / \mathrm{s}$ at $2.8 \mathrm{~K}$. In terms of atomization, this decrease in velocity is only partly compensated by the increase of the vapor density. Indeed, atomization is driven by the vapor kinetic energy density, which scales as $W_{\text {line }}^{2} / \rho_{\mathrm{v}}$, hence decreases with increasing temperature. This explains why atomization of normal liquid could not be obtained in the first experiments [8] with an injected liquid mass flow rate of $7 \mathrm{~g} / \mathrm{s}$ (correspond-

\footnotetext{
${ }^{4}$ In contrast to the LHC case, the mass flow rate of vapor is essentially constant along the line, as the distributed heat losses are less than $10 \mathrm{~W}$, much smaller than the typical heating powers applied in our studies $(100-360 \mathrm{~W})$.
} 
ing to a maximal $W_{\text {line }}=150 \mathrm{~W}$ ). This was one of the motivations [23] for increasing this mass flow up to $20 \mathrm{~g} / \mathrm{s}$ in the present experiments, allowing to compare the heat transfer for superfluid and normal helium in similar conditions of atomization [24, 25].

In the next sections, we describe the different probes that we used to characterize the atomized mist, the liquid film, and the thermal transfer. Previous measurements in a $22 \mathrm{~m}$ long pipe [6] have shown that, below $V_{\mathrm{v}}=7 \mathrm{~m} / \mathrm{s}$, the thermal transfer is identical $10 \mathrm{~m}$ and $20 \mathrm{~m}$ away from the inlet, suggesting that, for these velocities, the spray develops over a distance shorter than $10 \mathrm{~m}$. In the present experiments, our probes are located in the last two meters of the pipe (figure 1), in order to ensure that the observed spray is, if not fully developed, as developed as possible above $7 \mathrm{~m} / \mathrm{s}$.

\subsection{Optical characterization of the helium mist}

We characterize the helium mist by light scattering measurements performed in a glass portion of the pipe. As described in refs. [7, 25, 26, 27, 28], we use two differents methods. Quantitative (photometric) analysis of CCD pictures gives a time-averaged map of the local interfacial area of the droplets across the whole pipe cross-section. A Phase Doppler Particle Analyzer (PDPA) measures the size and axial velocity distributions of the droplets at the intersecting point of two laser beams.

The angular dependence of the scattered light close to the forward direction, measured in section (5) of the pipe [7, 28] (figure 1), shows that the droplets generated by the atomisation process are spherical and larger than several micrometers in diameter, so that the scattered light intensity measures the local interfacial area $\Sigma$ of the droplets [25, 28]. The distribution of $\Sigma$ across the pipe cross-section is then obtained by illuminating the pipe with a laser sheet propagating horizontally perpendicular to the pipe and imaging the mist with a CCD under a $15^{\circ}$ angle with respect to the direction of propagation of the laser sheet. Alternatively, we can also measure the profile of $\Sigma$ along the vertical diameter by shining a laser beam propagating vertically from the top to the bottom of the pipe and imaging the light scattered at $90^{\circ}$.

Individual snapshots taken at a rate of one frame per second reveal significant fluctuations of the droplets interfacial density. The fluctuation level, which does not depend on the exposure time between $3 \mathrm{~ms}$ and $30 \mathrm{~ms}$, is 
uncorrelated between successive images ${ }^{5}$, and is typically 15 to $25 \%$ of the average density, depending on physical conditions and on the position within the pipe cross-section. In order to reduce the noise, we average several tens of such snapshots. The intensity of the averaged pictures is then reproducible within several \%. This is comparable to the day-to-day reproducibility for given physical conditions.

Figure 2 shows averaged pictures for the laser sheet and the vertical laser beam, for an intermediate and a large vapor velocity, and the corresponding profiles of the interfacial density $\Sigma$ along the vertical tube diameter (see ref. [25] for details). When the velocity increases, the mist becomes denser, due to the increased atomisation, and less stratified, due to the larger turbulent diffusivity. Unexpectedly, the scattered intensities at $15^{\circ}$ (sheet illumination) and $90^{\circ}$ (vertical laser) give different values for the interfacial density near the bottom of the pipe. As discussed in ref.[25], this difference could result from multiple scattering effects, which mainly affect the intensity scattered at small angles. In any event, in the central region of the pipe where the local probes are located, the laser sheet gives values of $\Sigma$ comparable to those obtained from the vertical laser. This validates its use to determine the transverse structure of the droplets distribution.

Parallel to these global measurements, we also measure the diameter and velocity of the individual droplets using a Phase Doppler Particle Analyzer (PDPA) [7, 26]. The two beams of the PDPA are in a horizontal plane so that only the axial component of the velocity is measured. In most cases, measurements were only performed on the pipe axis, but the vertical and radial dependence of the droplets distribution was measured for several flow conditions $[27,29]$. We typically find a nearly exponential distribution of diameters $d, P(d) \propto \exp \left(-d / d_{0}\right)$, with $d_{0}$ ranging from 20 to $40 \mu \mathrm{m}$, depending on hydrodynamic conditions [29]. The velocity distribution is the same for all droplets, or droplets of a given diameter only. This shows that the droplets inertia is small enough for the droplets to follow the turbulent flow. Moreover, assuming the turbulence to be isotropic, the fluctuations of the velocity field should be comparable for all components. Hence, we can expect that the width $\delta v_{r . m . s .}$ of the axial velocity distribution gives an estimate of the droplets transverse velocity. This width is found between $5 \%$

\footnotetext{
${ }^{5}$ This indicates that the fluctuations of the mist density are temporally correlated over a time between $30 \mathrm{~ms}$ and $1 \mathrm{~s}$.
} 

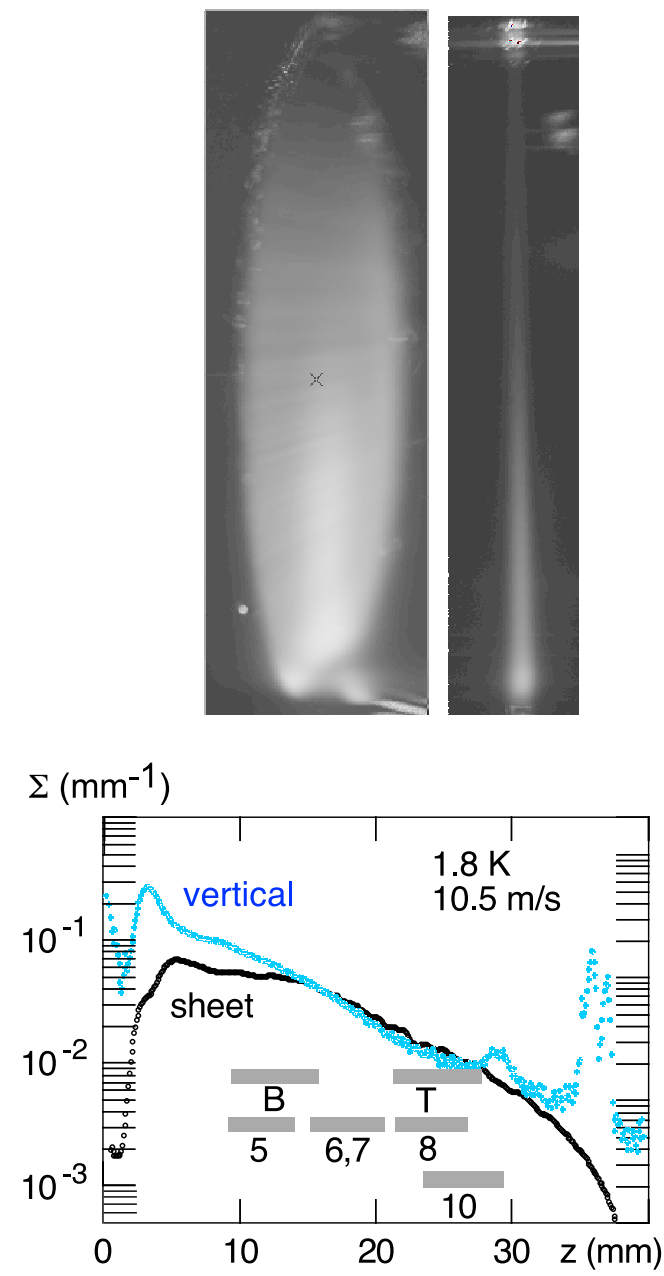
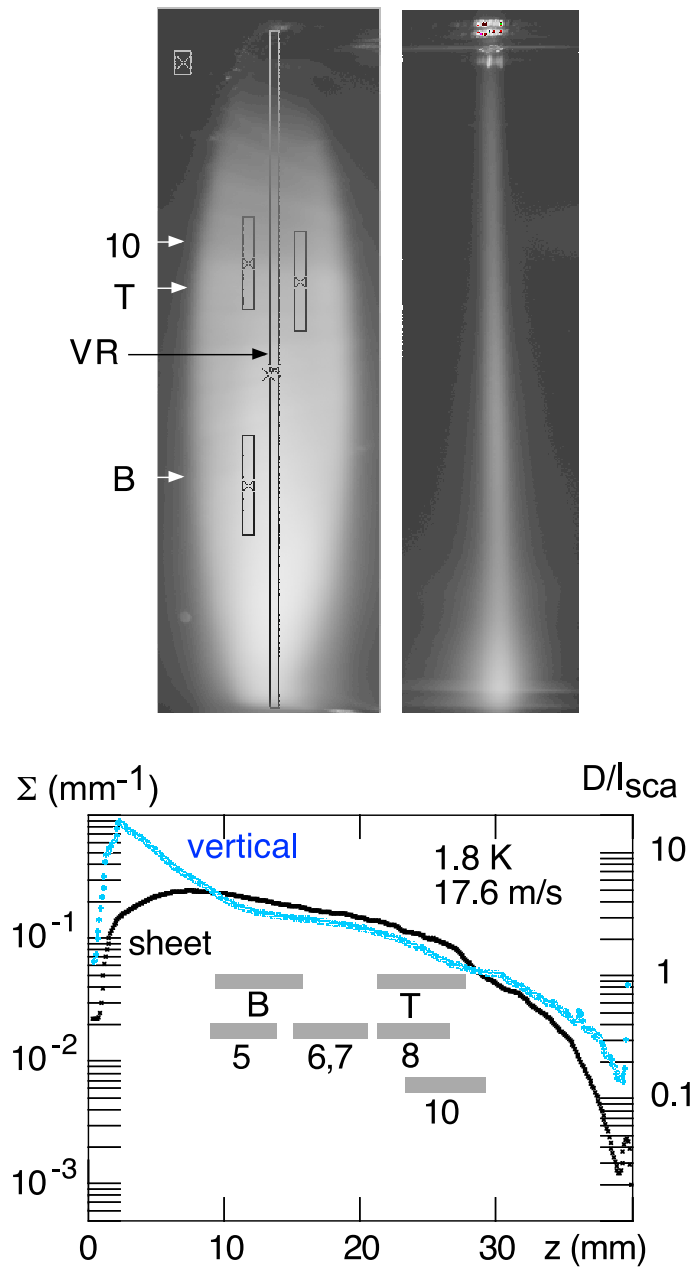

Figure 2: Vertical distribution of the mist for two vapor velocities $(10.5 \mathrm{~m} / \mathrm{s}$ and $17.6 \mathrm{~m} / \mathrm{s}$ on the pipe axis), and identical temperature $(1.8 \mathrm{~K}$ ) and liquid level (corresponding to $20 \%$ of the pipe perimeter). Averaged pictures (logarithmic grey scale) taken with both the laser sheet and the vertical laser illumination are shown. The exposure time is reduced from 30 to $10 \mathrm{~ms}$ for the sheet illumination at $17 \mathrm{~m} / \mathrm{s}$. The mist is denser and less stratified at large velocity. This is quantified by converting the grey level (i.e. the scattered intensity from the laser sheet) along a vertical diameter (VR), into the interfacial density $\Sigma$ transported by the droplets (left scale). The right scale corresponds to $D / l_{\text {sca }}$, the ratio of the pipe diameter to the light scattering mean free path, which measures the importance of multiple scattering [25]. Measurements using the vertical laser beam give a similar profile in the central part of the pipe, where the thermal (B: bottom ; T: top) and capacitive probes are located (figures 3 and 5). 


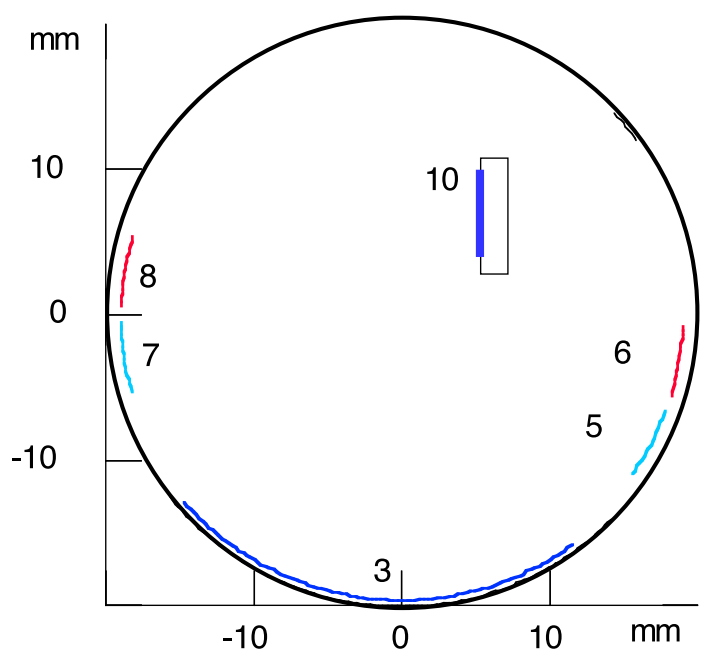

Figure 3: Angular location of the interdigitated capacitors. Capacitor 3 is used to measure the bulk liquid level, capacitors 5 to 8 , and the suspended capacitor 10, located downstream, measure the local film thickness.

and $10 \%$ of the axial velocity, which is typical for developed turbulence.

\subsection{Capacitive film detection}

We use interdigitated capacitors, evaporated on flexible Kapton foils [9, 30], to measure the thickness of the helium film locally deposited by the droplets. A numerical simulation of the electrostatic problem [30] shows that, for uniform thicknesses smaller than the gap between electrodes (30 or $50 \mu \mathrm{m}$ ), the capacitance changes linearly with thickness, with a slope which depends on the electrostatic characteristics of the device. In this range, this allows us to convert the measured capacitance into a film thickness (averaged over the probe area). Based on this principle, different capacitors glued onto the walls of the pipe (figure 3) measure the height dependence of the film thickness. In addition, a planar capacitor is suspended by Kevlar wires across the pipe (at the same height as the top thermal probe discussed below). Unlike the capacitors on the pipe walls, it is insensitive to waves of the liquid level, or to the film flowing from higher elevations. It can thus be expected to measure a film thickness directly correlated to the locally deposited droplets flux.

A similar capacitor is glued on the bottom part of the pipe (covering $25 \%$ of its perimeter). In contrast to the other capacitors, it is partly covered by 


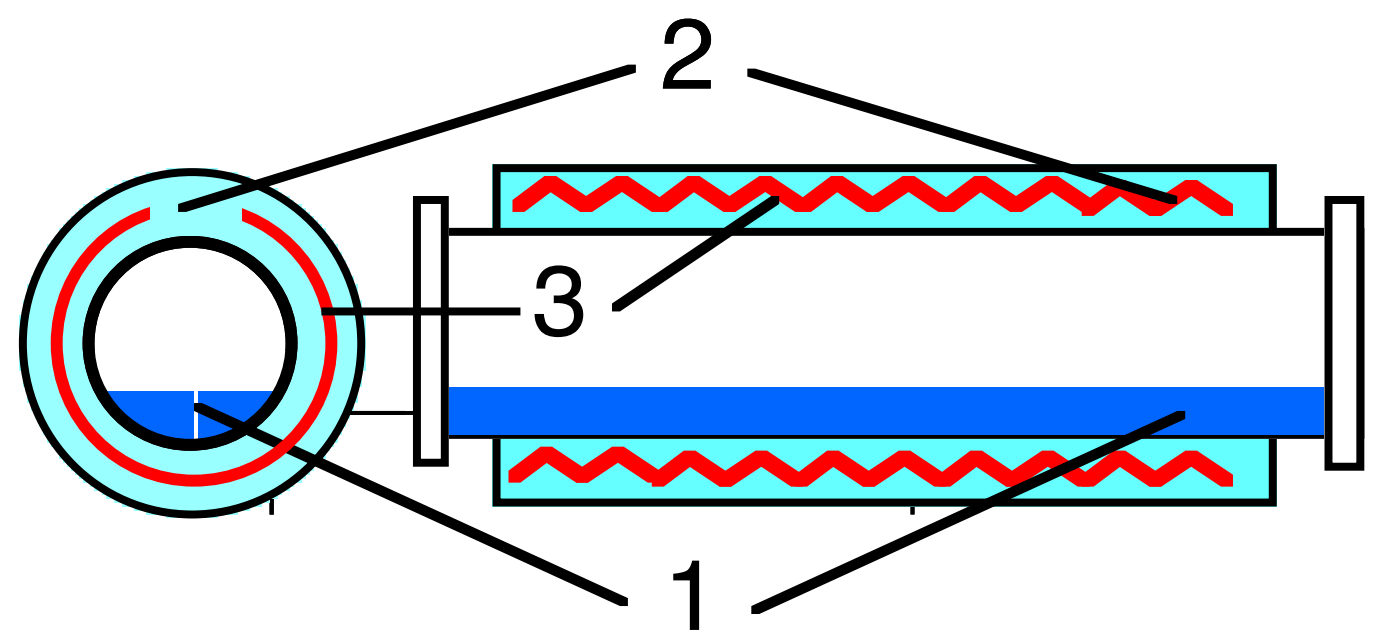

Figure 4: Characterization of the global heat transfer using the Kapitza box. The temperature difference between pressurized (2) and saturated (1) helium is measured as a function of the power $W_{K}$ applied to the heater (3).

the bulk liquid flowing in the pipe. The excess capacitance with respect to the dry state is then proportional to the area covered by this bulk liquid ${ }^{6}$. From this area, we infer the bulk liquid level [30, 31].

\subsection{Global heat transfer}

The global capability of the diphasic flow to extract heat from the pipe walls is measured by a set-up reproducing the LHC situation (inset of figure 4). This so-called Kapitza box [32] is made of two concentric tubes (40 $\mathrm{mm}$ and $76 \mathrm{~mm}$ in diameter) $40 \mathrm{~cm}$ long. The $1 \mathrm{~mm}$ thick inner tube is in copper and contains the diphasic flow, in continuity with the stainless steel pipe. The space between the two tubes, filled with superfluid liquid at 1 bar, plays the role of the LHC magnet bath. An annular heater simulates the heat load dissipated by the magnet, and two thermometers allow to measure the temperature increase of the pressurized liquid helium as a function of this heat load, up to typically $10 \mathrm{~W}$. In this range, the heat transport in the superfluid follows the Görter-Mellink regime, and the pressurized liquid can be considered isothermal on the scale of the temperature difference between

\footnotetext{
${ }^{6}$ The correction due to the film deposited above the bulk liquid level is small thanks to the large gap of this capacitor $(100 \mu \mathrm{m})$.
} 


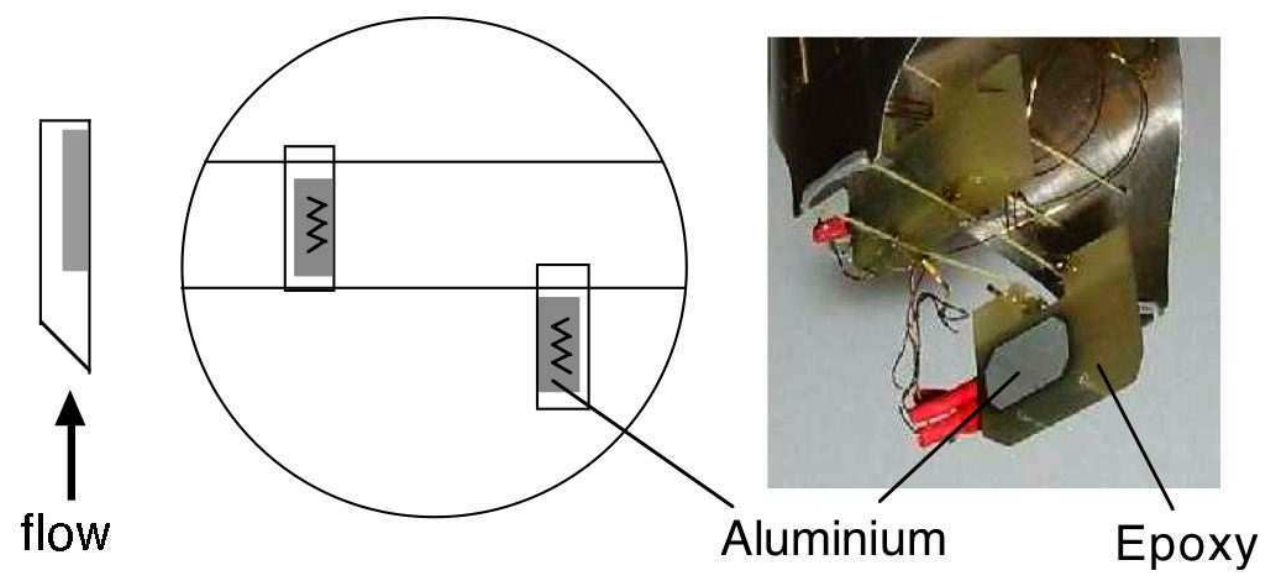

Figure 5: Location of the thermal probes in the pipe cross-section. The aluminum part of the bottom and top probes is shaded in grey. The left figure shows the top probe seen from above.

the bath and the pipe. The latter involves the Kapitza resistance between the pressurized bath and the copper tube, the thermal resistance of the copper, and the Kapitza resistance between the copper and the saturated liquid covering the walls (the heat exchange with the vapor is negligible). The thermal conductivity of the copper tube, measured independently, is of order $10 \mathrm{~W} / \mathrm{K} . \mathrm{m}$ at $1.8 \mathrm{~K}$, small enough for the heat flux inside the copper to be essentially radial, and large enough for the Kapitza resistance to be the dominant contribution $(\approx 80 \%)$ to the global resistance. The global heat exchange coefficient is then nearly proportional to the wetted area of the pipe inner wall. The coefficient of proportionality, i.e. the heat exchange for $100 \%$ coverage, was separately determined [8] to be about $100 \mathrm{~W} / \mathrm{K}$ at $1.8 \mathrm{~K}$.

In typical conditions, the thermalization time of the pressurized bath is of order 20-30 s, as determined by the heat exchange and the pressurized superfluid thermal capacity. The heat transfer in the atomized regime is characterized by applying successive constant powers to the heater in the range $0.25 \mathrm{~W}$ to $10 \mathrm{~W}$, and measuring the temperature of the pressurized bath, once it has come to equilibrium.

\subsection{Local heat transfer}

The Kapitza box measures the global heat transfer between the diphasic flow and the walls, which is the relevant quantity for cryogenic applications. 
However, it integrates various contributions (from the bulk liquid, from the film possibly deposited by waves, and from the film deposited by the stratified mist), and does not allow to separate the contribution of the mist to the thermal transfer. On that purpose, we use two thermal probes at two different heights in the last portion of the pipe (figure 5). The probes are suspended using Kevlar wires thin enough for the contribution of the Rollin film to be negligible. Each probe consists in a pure (99.999\%) aluminum polished plate (6 mm high, $10 \mathrm{~mm}$ long, and $3 \mathrm{~mm}$ thick) embedded in epoxy so as to leave apparent only one face. A heater and a thermometer at two different locations inside the aluminum plate allow to measure its temperature increase as a function of the applied power. The epoxy facing the vapor flow is wedged, so as to deflect the axial flow of droplets and warrant that droplets impinge onto the probe mainly due to their transverse, rather than axial, motion. We therefore expect the probes to be cooled by the mist only through this transverse motion, as is the case for the pipe walls. The aluminum thermal conductivity is large enough for the plate to be isothermal. The thermal exchange between the plate and liquid helium was separately measured by immersing the probe into a superfluid bath. The results are consistent with a Kapitza resistance of order $1.3 \mathrm{~K} . \mathrm{cm}^{2} / \mathrm{W}$ at $1.8 \mathrm{~K}$, varying as $1 / T^{2}$. No film boiling was detected up to $2 \mathrm{~W} / \mathrm{cm}^{2}$.

\section{Results}

\subsection{Atomization}

Let us briefly describe the evolution of atomization with the physical conditions, as characterized from our optical measurements, focusing on its relevance to the present study. First, in all conditions, the mist is stratified, with a nearly exponential profile of the interfacial density $\Sigma$. The characteristic stratification height increases with the vapor velocity, but does not depend on the liquid level, and only marginally on the vapor density (i.e. the temperature, since the vapor is saturated). In the simple model of stratification described in $\S 3$, the characteristic height decreases with the droplets size, and increases with the vapor viscosity and the droplets diffusivity due to turbulence. The observed features are qualitatively consistent with such a model, as the droplets size is expected to decrease, and their diffusivity to increase, with increasing vapor velocity. In contrast to the stratification height, the global level of atomization, as measured by $\Sigma$, increases with any of the above three physical parameters, keeping the two others fixed. The 
increase of atomization with the level of liquid in the pipe at least partly reflects the larger area of the bulk liquid-vapor interface. In the superfluid phase, the effect of the vapor density and temperature follows from the corresponding increase in the vapor kinetic energy. As compared to the superfluid, atomization is stronger, for a given vapor velocity, in the normal fluid. It is not clear whether this increase only results from the large increase of the vapor density between $2 \mathrm{~K}$ and $2.25 \mathrm{~K}$ (the largest and the smallest temperatures studied in the superfluid and normal fluid, respectively), or whether it involves a specific effect of superfluidity on atomization.

In any case, the fact that the global degree of atomization, but not the stratification height, increases with the liquid level allows us, by adjusting the latter, to compare situations at different temperatures, but with similar spray characteristics in terms of $\Sigma$ and axial velocity. This will enable us to compare the efficiency of spray cooling, as measured by the suspended thermal probes, between the normal and the superfluid phases.

We finally discuss the transverse structure of the mist. Optical observations reveal a complex dependence of this structure, possibly reflecting changes in the flow pattern. For most of our experimental conditions, at a given height in the pipe, the mist is denser on the pipe median plane than close to the walls, except in the top most part of the pipe where the situation is reversed (figure 6a). The interfacial density $\Sigma$ at the height of the bottom thermal probe can thus be nearly twice as large on the pipe median plane than close to the walls. In contrast, for a very low liquid level (figure 6b), in the bottom half of the pipe, the droplets density is larger close to the walls. This horizontal structure of the mist should be kept in mind when comparing measurements using the suspended probes to those implying the pipe walls (global heat transfer, film thickness).

\subsection{Film thickness}

Simultaneously to the observation of a mist of droplets, the different capacitors evidence the presence of a liquid film on the pipe walls and on the suspended probe. The thickness of this film, which ranges from several tenths of micrometers to ten micrometers, decreases from the bottom to the top of the pipe, and increases with the degree of atomization. The correlation between $\Sigma$, the local interfacial density of the mist, and the film thickness is shown in figure 7 for a large set of atomization conditions, corresponding to different liquid levels, vapor velocities, and temperatures. It demonstrates that the film formation results from droplets deposition. Note that, at a 

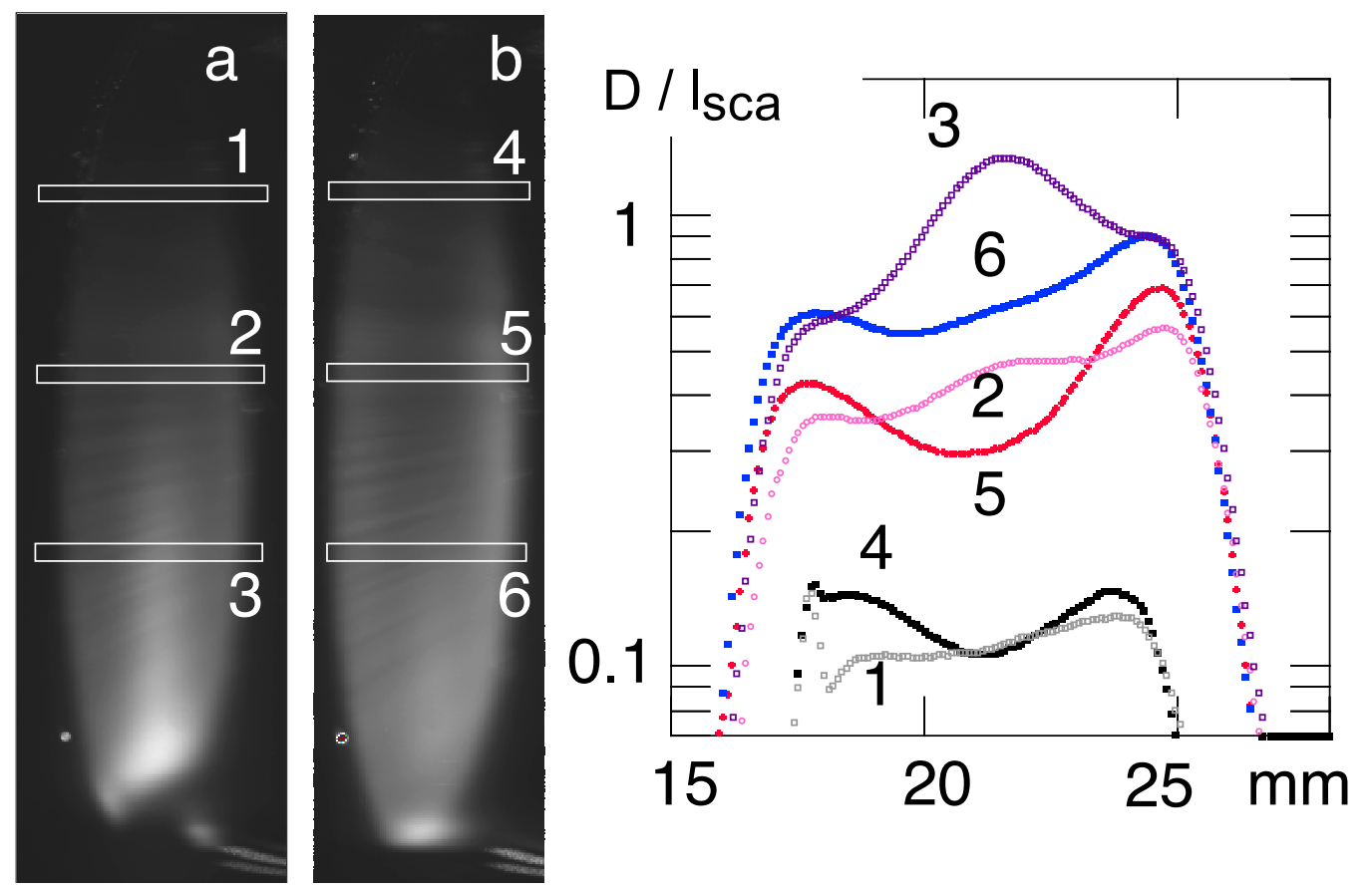

Figure 6: The transverse structure of the droplets distribution depends on the physical conditions. This should be taken into account when comparing measurements using the suspended probes to those implying the pipe walls. The pictures (in grey level, with increased contrast) show the mist for (a) high liquid level (24\% of total perimeter) at $1.85 \mathrm{~K}$ and an averaged velocity of $10.2 \mathrm{~m} / \mathrm{s}$ and; (b) low liquid level (14\% of total perimeter) at $2 \mathrm{~K}$ and an averaged velocity of $12.1 \mathrm{~m} / \mathrm{s}$. The horizontal profile of the interfacial density at three different heights, the two lower corresponding to the position of the suspended thermal probes, is shown in the plot. The asymetry between left and right is due to a perspective effect (in each rectangle, the height decreases from left to right), combined with the stratification. For (a), the droplets interfacial density is maximal on the pipe median plane, except in the pipe top most part. In contrast, for (b), it is maximal near the walls.

given $\Sigma$, superfluidity has only a limited influence on the film thickness. We will come back to this point in section $\S 6.2$. Figure 7 also shows that the thickness increases non linearly with $\Sigma$. We will see below that this reflects the fact that the film drainage, which balances the incident liquid flux, depends non linearly on the film thickness. The data for the different probes do not collapse on a single curve. This could be due to the fact that 


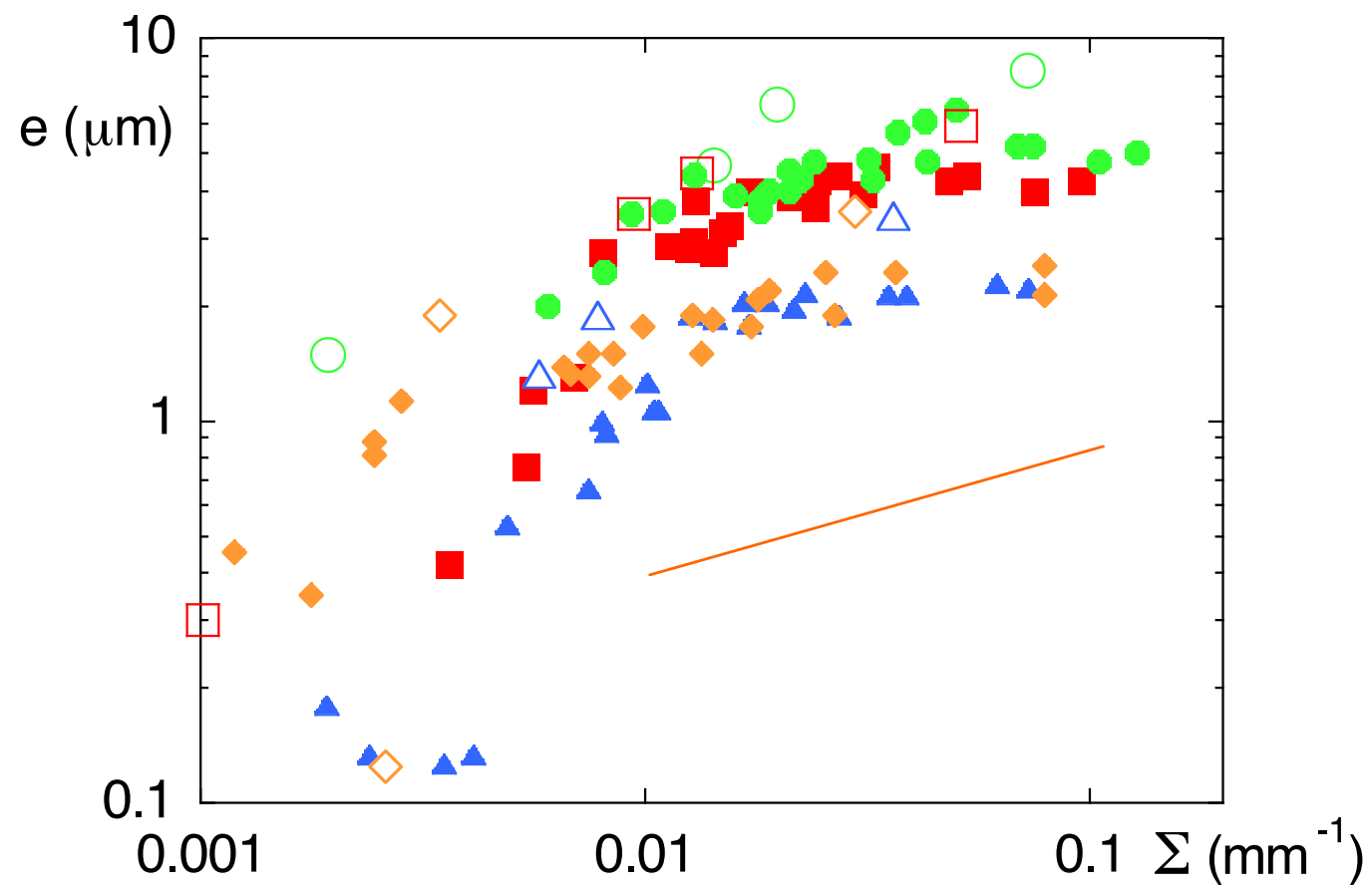

Figure 7: Dependence of the thickness (assumed to be uniform) of the film covering the different capacitive probes on the local mist interfacial density $\Sigma$. Closed symbols: superfluid phase; $\bullet$ : bottom probe (5 in figure 3 ); $\mathbf{\square}$ : probe 6 (results for 7 are similar); $\mathbf{\Delta}$ : top probe (8); $\$$ : suspended probe (10). Larger open symbols correspond to points in the normal phase of liquid. $\Sigma$ is measured using the laser sheet. For a given probe, the film thickness mainly depends on $\Sigma$. It tends to be larger in the normal phase. The straight line corresponds to the $1 / 3$ power law behaviour expected if the incident volumic flux is proportional to $\Sigma$ only (see $\S 6.2$ ). 
the incident flux does not only depend on $\Sigma$, but also on the average size and impact velocity of droplets, which can vary within the pipe cross-section.

\subsection{Global heat transfer}

The formation of a liquid film increases the wetted surface and improves the thermal contact between the pipe walls and the diphasic flow. This is shown in figure 8, which compares the thermal transfer measured by the Kapitza box in the non-atomized and atomized regimes. Although the bulk liquid level is similar in both cases, the heat transfer is much more efficient in the second case. However, the temperature difference increases faster than the applied heat flux. This implies that the deposited film is progressively evaporated as the heat load increases. In the next section, we direcly evidence this phenomenon using the suspended thermal probes.

\subsection{Cooling power of the mist}

Figure 9 provides clear evidence for the critical heat flux phenomenon. It shows the temperature of the top probe as a function of the applied power for different vapor velocities at a constant liquid level and for a temperature of $1.8 \mathrm{~K}$, i.e. in the superfluid phase. Except for the lowest vapor velocity of $7 \mathrm{~m} / \mathrm{s}$, a linear regime is observed at low applied power, with a slope equal to that found for the probe totally immersed in superfluid helium. This shows that the probe is totally covered by a liquid film, in agreement with the conclusion drawn from the results for the suspended capacitive probe. This regime extends up to a critical threshold which increases with the vapor velocity, beyond which the probe temperature rises abruptly ${ }^{7}$. At $7 \mathrm{~m} / \mathrm{s}$ (resp. $10 \mathrm{~m} / \mathrm{s}$ ), the slope above the threshold is about $90 \mathrm{~K} / \mathrm{W}$ (resp. $60 \mathrm{~K} / \mathrm{W})$. Such thermal resistances are consistent with that expected from thermal exchange with the turbulent vapor, about $200 \mathrm{~K} / \mathrm{W}$ at $1.8 \mathrm{~K}$ and $10 \mathrm{~m} / \mathrm{s}^{8}$. This confirms that the threshold corresponds to evaporating the

\footnotetext{
${ }^{7}$ Note that the initial linear regime extends up to the threshold. This contrasts with the case of classical fluids, such as water, where the thermal resistance decreases as the threshold is approached, probably due to vapor nucleation inside inside the film. Such a phenomenon cannot occur here since the superfluid film is isotherm.

${ }^{8}$ Thermal exchange with the turbulent vapor is given by $h=N u . \kappa / l$, where $h$ is the thermal exchange coefficient, $\kappa$ the vapor thermal conductivity, $l$ the length of the active probe length, and $N u$ the Nusselt number, estimated by the Colburn formula, $N u=0.023 \cdot \operatorname{Re}^{0.8} \cdot \operatorname{Pr}^{1 / 3}$, with $\operatorname{Re}$ and $\operatorname{Pr}$ the Reynolds and Prandtl numbers.
} 


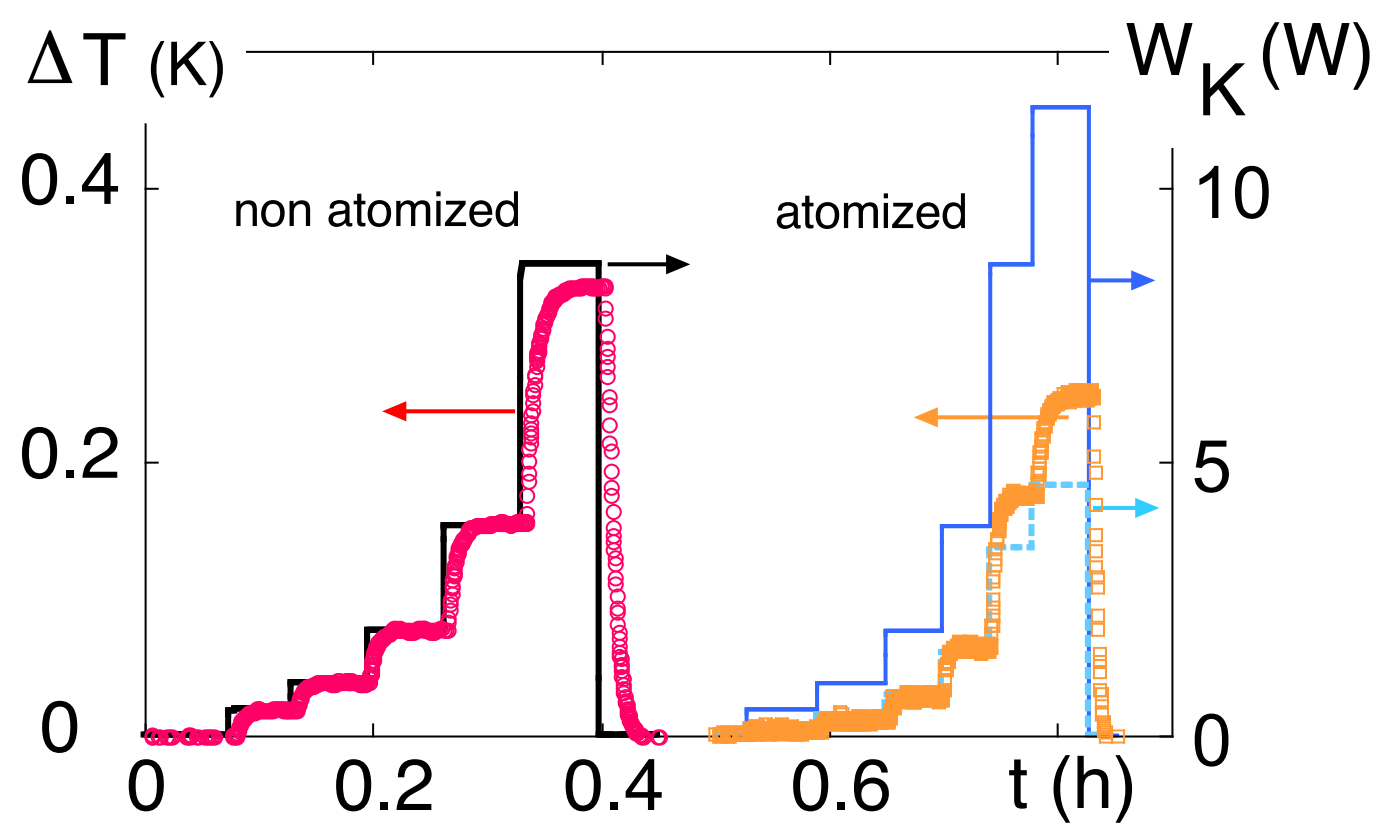

Figure 8: Characterization of the global heat transfer using the Kapitza box. The temperature difference between pressurized and saturated helium is shown as a function of the power $W_{K}$ for non-atomized and atomized conditions and similar bulk levels $(\mathrm{T}=1.8 \mathrm{~K}$ for the saturated liquid, applied power $W_{\text {line }}=27 \mathrm{~W}$ and $180 \mathrm{~W}$ corresponding to $V_{\mathrm{v}}=3$ and $15 \mathrm{~m} / \mathrm{s}$ on the pipe axis, injected flow rate $\dot{m}=4$ and $12 \mathrm{~g} / \mathrm{s}$, corresponding to a wetted perimeter by the bulk liquid of $23 \%$ and $20 \%$ of the total perimeter). Although taken on different days, the two data sets are plotted on the same time scale for comparison. In the non atomized state, the temperature increases linearly with the applied power, except at large power due to the decrease of the Kapitza resistance at large temperatures. In the atomized state, the temperature rise is smaller than in the non atomized state, due to the film deposited by the atomized droplets, but increases faster than the applied power, because the film is evaporated by the applied heat. This is directly seen by comparing the temperature increase with the dotted curve, which shows the applied power for the atomized case, rescaled such as to coincide with the temperature rise at $t=0.7 \mathrm{~h}$. 


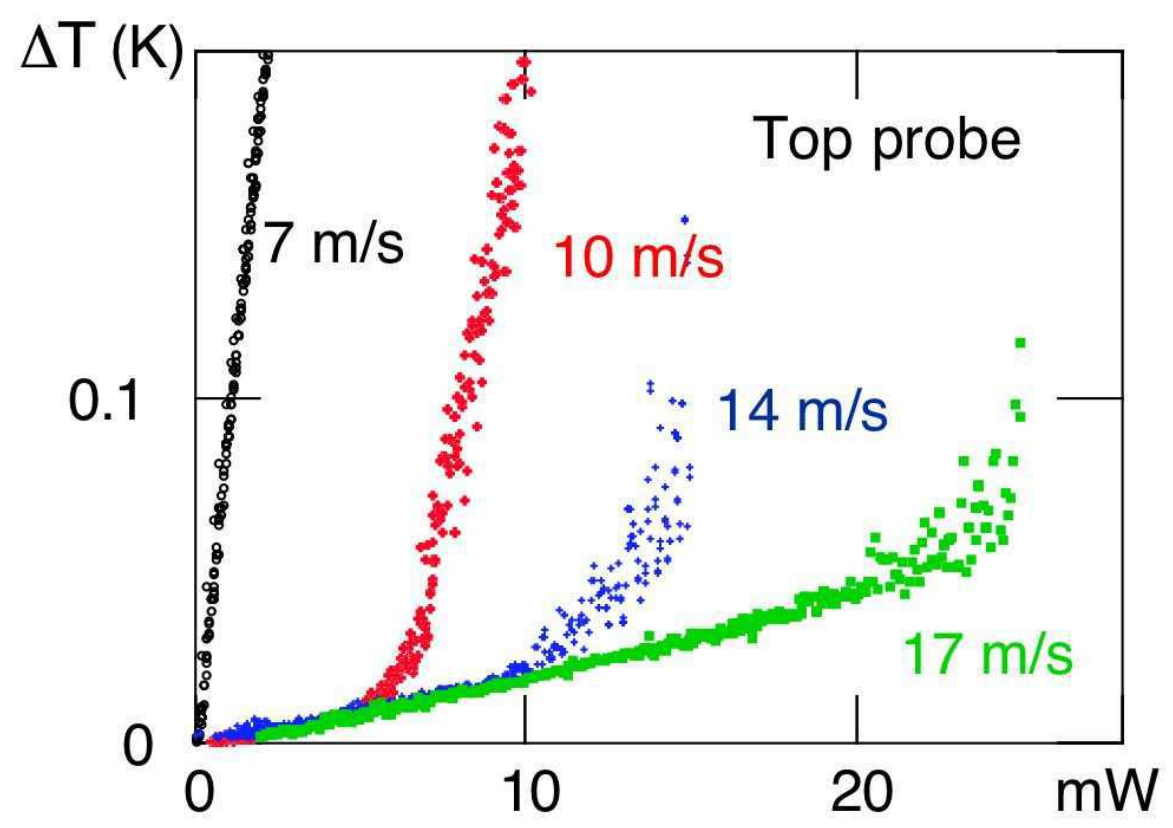

Figure 9: Temperature of the top probe as a function of the applied power in $\mathrm{mW}$, for different flow velocities $(\mathrm{T}=1.80 \mathrm{~K}$, liquid height $\approx 3.5 \mathrm{~mm}$ ). The temperature is controlled by the Kapitza resistance between the aluminum plate and the deposited liquid film below a critical heat flux, and by exchange with the turbulent vapor above. Like the mist density, the critical heat flux, which corresponds to full vaporization of the deposited liquid flow, increases with the vapor velocity. 
liquid flux deposited by the droplets, hence to the CHF. The curve at $10 \mathrm{~m} / \mathrm{s}$ shows that the behaviour above the threshold remains linear up to large temperature differences. This implies that the cooling power due to the mist vaporization does not decrease as the temperature of the probe increases, hence that the interaction of droplets with the wall is unaffected by the wall temperature, at least in the explored range. In other words, we operate in a regime of cold walls, with no Leidenfrost (calefaction) phenomenon.

The same behaviour is observed at any temperature in the superfluid phase, except that the initial slope decreases with increasing temperature, in agreement with the measurements performed with the immersed probes. The situation is different in the normal phase. This is clearly seen by comparing in figure 10 the behaviour of the thermal probes for two temperatures of the normal fluid, $2.24 \mathrm{~K}$ and $2.33 \mathrm{~K}$, to that observed in the superfluid phase, for equivalent spray properties. As shown by figure 11, each normal fluid condition has a close superfluid equivalent in terms of the profile of the interfacial density along a vertical diameter, the droplets size distribution and the vapor velocity distribution on the pipe axis. The liquid flux impinging on the suspended thermal probes should then be comparable for each of these couples of points ${ }^{9}$.

Figure 10 shows that the behaviour in the normal phase differs from that in the superfluid phase in three respects. First, the temperature signal is noisier. By averaging over a number of sweeps, we can still define a threshold below which the temperature rise varies linearly with the applied heat flux as well as a slope for the initial linear behaviour. However, in contrast to the superfluid case, some measured data points lie above the linear behaviour below the threshold or, alternatively, follow this behaviour above it (see in particular figure 10d). This last point suggests that the noise below the threshold may result from the larger fluctuations of the incoming flux of droplets $^{10}$, rather than from incomplete spreading of the normal fluid droplets on the heated metal plate. Secondly, while the Kapitza resistance between the metal plate and the film should decrease with increasing temperature

\footnotetext{
${ }^{9}$ Refering to $\S 3$, the impact velocity could be reduced with respect to $\delta v_{\text {r.m.s. }}$ due to inertia effects and interaction between droplets. However, because $\Sigma$ and $d_{0}$ are similar in both cases, the reduction should be similar as well.

${ }^{10}$ The CCD images show that the interfacial density fluctuates much more in the normal phase than in the superfluid phase, at least partly reflecting the difficulty to regulate the liquid level in this phase.
} 

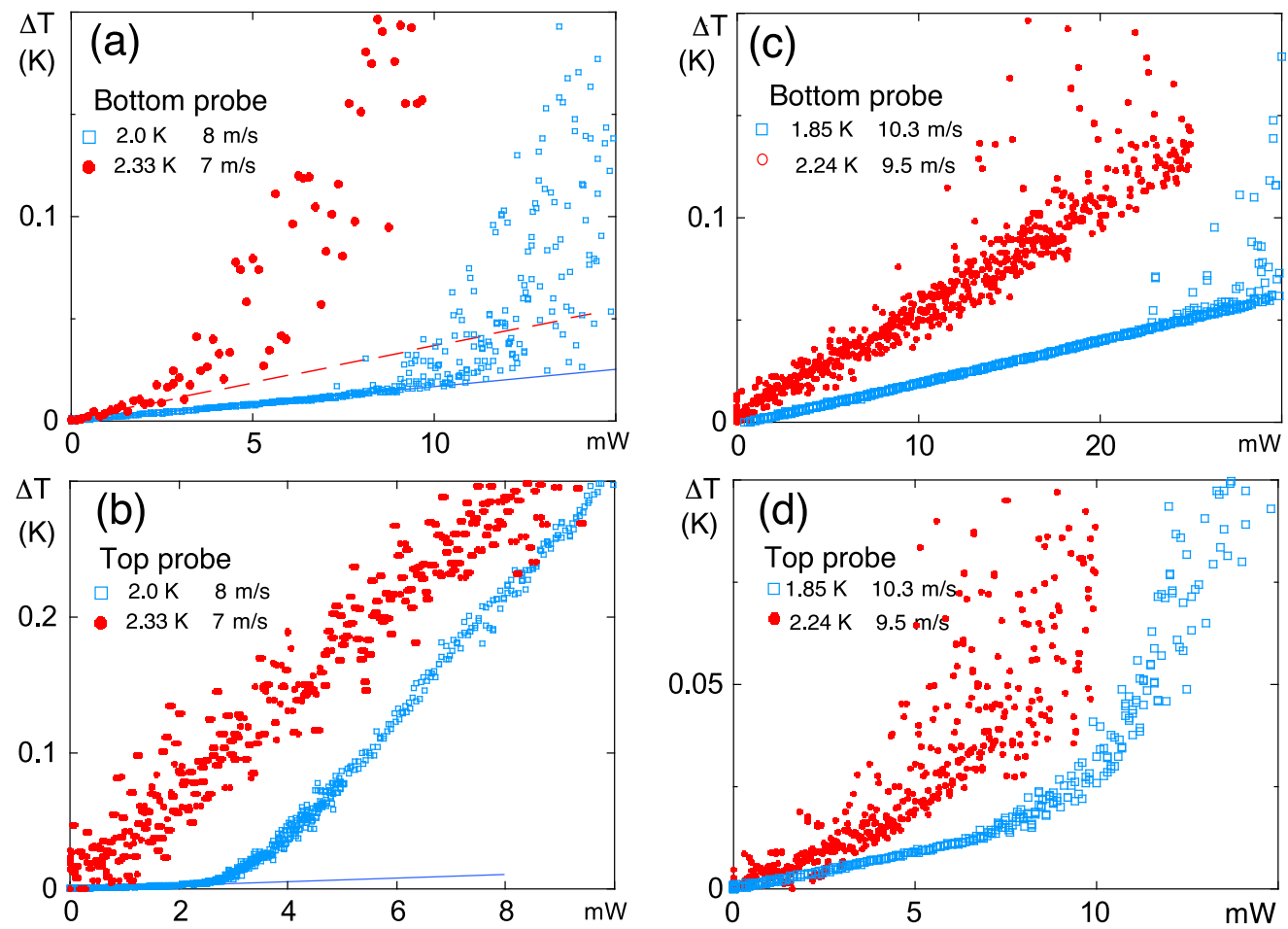

Figure 10: Superfluidity affects the cooling power of the mist. Thermal characteristics of the bottom $(\mathrm{a}, \mathrm{c})$ and top $(\mathrm{b}, \mathrm{d})$ thermal probes in the superfluid $(\mathrm{SF})$ and normal $(\mathrm{N})$ phases for similar spray conditions. (a,b): Temperatures $2 \mathrm{~K}$ and $2.33 \mathrm{~K}$, average vapor velocities of $8(\mathrm{SF})$ and $7 \mathrm{~m} / \mathrm{s}(\mathrm{N})$; (c,d) Temperatures $1.85 \mathrm{~K}$ and $2.24 \mathrm{~K}$, average vapor velocities of $10.3(\mathrm{SF})$ and $9.5 \mathrm{~m} / \mathrm{s}(\mathrm{N})$. The straight lines in (a) and (b) correspond to the linear extrapolation of the low heating power regime. For each pair of conditions, the liquid level is adjusted between 3.8 and $4.8 \mathrm{~mm}$ so as to obtain similar spray characteristics in the normal and superfluid phases (see figure 11). 

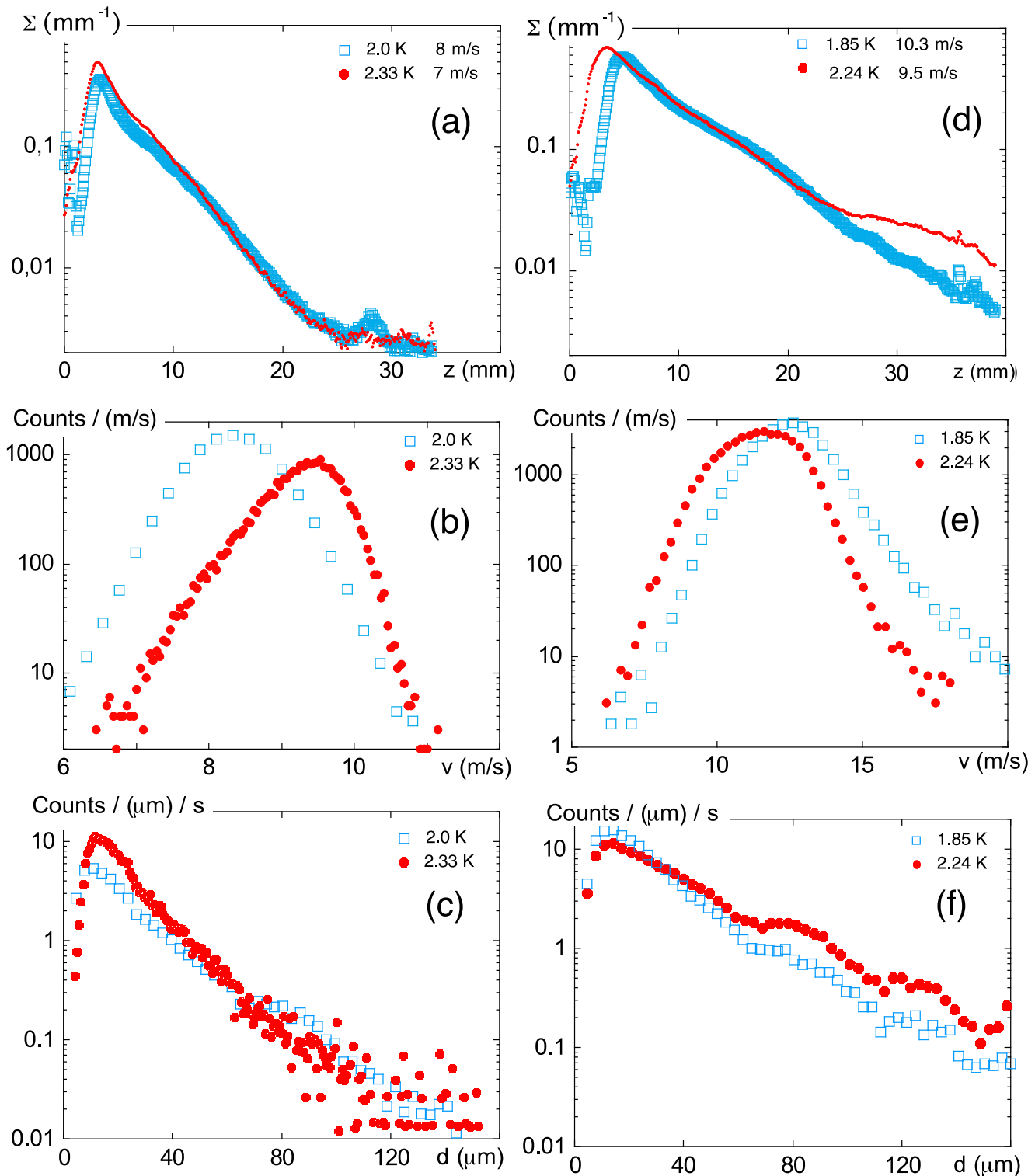

Figure 11: Spray characteristics for the two pairs of data sets shown in figure 10: (a,d) Vertical profile of the scattered intensity, as measured with the vertical laser ; $(b, e)$ : Histograms of the axial velocity on the pipe axis ; $(c, f)$ : Droplets size distribution on the pipe axis. (a,b,c): Temperatures $2 \mathrm{~K}$ and $2.33 \mathrm{~K}$, average vapor velocities of 8 (SF) and $7 \mathrm{~m} / \mathrm{s}(\mathrm{N}) ;(\mathrm{d}, \mathrm{e}, \mathrm{f})$ Temperatures $1.85 \mathrm{~K}$ and $2.24 \mathrm{~K}$, average vapor velocities of 10.3 (SF) and $9.5 \mathrm{~m} / \mathrm{s}(\mathrm{N})$. 
(which is indeed the case in the superfluid phase, as shown by comparing $1.85 \mathrm{~K}$ and $2 \mathrm{~K}$ in figure 10 ), the initial slope is larger in the normal phase than in the superfluid one. The corresponding extra contribution to the thermal resistance, which ranges from 2 to $4 \mathrm{~K} / \mathrm{W}$, depending on the probe and on the temperature of the normal fluid, is likely due to the poor thermal conductivity of the normal liquid. In this interpretation, it would correspond to a film thickness of 2 to $4 \mu \mathrm{m}$, a factor of two smaller than the value measured by the capacitive probes 5 and 10 for the same conditions ( 4 to $8 \mu \mathrm{m})$. Possible explanations for this factor of two discrepancy are a non uniform film thickness (the thermal resistance and the capacitance do not average the film thickness in the same way), or, in a similar way to classical liquids, boiling inside the liquid film. The fact that we do not observe, within the noise, a strong non linearity of the heat transfer below the threshold would rather favor the first interpretation. In any case, this result suggests that, below the threshold, the surface is fully covered by the normal liquid. The third, and most spectacular difference, is that the critical flux is significantly smaller in the normal state, and can even vanish for a small enough level of atomization (figure 10b).

This difference of behaviour implies that, when plotted against the interfacial density, the critical flux seems to extrapolate to a finite value at zero interfacial density in the superfluid phase, while this is not the case in the normal phase (figure 12a ). We believe that the excess cooling power in the superfluid phase is not due to a specific role of superfluidity in the spreading process, but rather to thermally driven superfluid mass transport from the probe cold epoxy surfaces to the heated metallic surface. For small heat fluxes, the liquid flux impinging on the whole probe is available for cooling, and we expect the critical flux to be proportional to the total probe area $S_{t}$ (about 6 times larger than the active area, counting only the lateral surfaces of the probe $\left.{ }^{11}\right)$. However, the lateral superfluid flow towards the active area must be limited by the critical velocity of the superfluid film. For large applied heat fluxes, we then expect the measured critical flux $W_{c}$ to be proportional to the active area $S_{a}$ only, plus a constant contribution $W_{\text {film }}$ proportional to the critical superfluid velocity, namely:

\footnotetext{
${ }^{11}$ The ratio $\frac{S_{t}}{S_{a}}$ could be larger than 6 , if some droplets impinge the front surface of the probe, despite its wedged shape.
} 

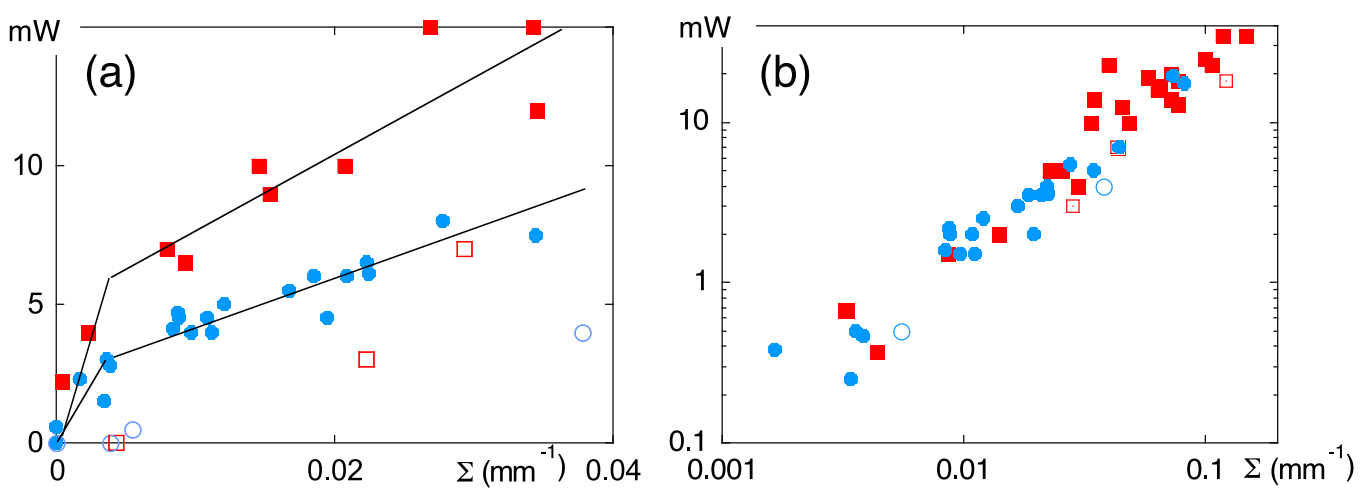

Figure 12: Critical heat flux $W_{c}$ for drying the top and bottom suspended probes, as a function of the interfacial area of the mist facing the probes, measured using the laser sheet. $\mathbf{\square}$ : Bottom thermal probe ; • Top bottom probe: Open and closed symbols refer to normal fluid and superfluid, respectively. (a) Linear plot of raw data for small interfacial densities. The straight lines show the behaviour expected from equations 1 and 2 , assuming the incident flux $\Phi$ to be proportional to $\Sigma$ and $\frac{S_{t}}{S_{a}}=6$; (b) Double logarithmic plot of the same data, after correcting $W_{c}$ for the contribution of lateral superflow. Within the dispersion, the global behaviour is consistent with a linear dependence on $\Sigma$ of $W_{c o r}$, the contribution of droplets impinging on the metallic part of the probe. $W_{c o r}$ is similar in the normal and superfluid phases, showing that the superfluid nature of the liquid droplets does not significantly improve their ability to cool the heated probes. 


$$
\begin{array}{cc}
W_{c}=L \Phi S_{t} & \left(W_{c}<\frac{S_{t}}{S_{t}-S_{a}} W_{f i l m}\right) \\
W_{c}=L \Phi S_{a}+W_{f i l m} & \left(W_{c}>\frac{S_{t}}{S_{t}-S_{a}} W_{f i l m}\right)
\end{array}
$$

where $\Phi$ is the incident liquid mass flux per unit probe area, and $L$ the latent heat. Accordingly, $W_{\text {film }}$ is estimated in figure 12a by linearly extrapolating a linear behaviour to zero interfacial density. This gives 5 and $2.5 \mathrm{~mW}$ for the bottom and top probes, respectively. These numbers can be compared to the cooling power of a saturated film, which we have directly estimated during the calibration experiment described in $\S 4.5$, by partly immersing $(\simeq 10-30 \%)$ the two thermal probes into a helium bath at $1.8 \mathrm{~K}$, and measuring their thermal characteristics. In this case, for applied powers larger than $10 \mathrm{~mW}$, the probe temperature increases linearly with the applied heat flux, but extrapolates to a finite power, of order 4-5 $\mathrm{mW}$, at zero temperature difference. In our interpretation, this extra-power is carried away by the continuous vaporization of the Rollin film covering the probe above the liquid free surface. The border of the aluminum plate being $\simeq 30 \mathrm{~mm}$ in length, $5 \mathrm{~mW}$ correspond to a critical flux transported by the film of order $1.5 \mathrm{~mW} / \mathrm{cm}$, i.e. roughly $5.10^{-4} \mathrm{~cm}^{2} / \mathrm{s}$. Although this number is five times larger than reported for the thermally [33] or gravitationally [34, 35] driven film flows on glass, the order of magnitude is similar, making our interpretation likely. The fact that $W_{\text {film }}$ for both probes is similar to the offset found in the calibration experiment supports our hypothesis that it is due to superfluid transport of the film ${ }^{12}$. Under this assumption, we can compute a corrected critical flux $W_{\text {cor }}$, corresponding to the liquid flux directly impinging on the probe active area, by inverting equations 1 and 2. Figure 12b shows how $W_{\text {cor }}$ depends on the interfacial density $\Sigma^{13}$. This correction makes the critical flux approximately linear in the interfacial density $\Sigma$ in a

\footnotetext{
${ }^{12}$ The origin of the smaller value of $W_{\text {film }}$ for the top probe, compared to the bottom one, is unclear. One possibility is that it results from the smaller thickness of the film outside the active probe area, due to the smaller impinging liquid flux.

${ }^{13}$ In this figure, in order to avoid the saturation at low height of $\Sigma$ measured using the laser sheet (fig. 2), we obtain $\Sigma$ close to the bottom probe using the vertical laser, and correcting for the lateral structure of the mist, as estimated using the laser sheet. However, the conclusion of a globally linear behaviour would not be affected by measuring $\Sigma$ from the laser sheet only.
} 
much broader range than in figure 12a. Moreover, the data in the normal phase now fall close to the superfluid corrected data. We conclude that the superfluid nature of the liquid droplets does not significantly improve their ability to cool the heated probes.

\section{Discussion}

\subsection{The impact velocity of droplets}

Figure $12 \mathrm{~b}$ can be used to estimate the impact velocity of droplets. Assuming the spray efficiency to be 1 (the incoming droplets do not bounce on the surface, but are fully evaporated), the incoming mass flux is given by

$$
\Phi_{i n c}=W_{\text {cor }} /\left(L . S_{a}\right)
$$

In our case of spherical droplets with an exponential distribution of diameters, the liquid volume fraction is $\Sigma d_{0} / 2$. Assuming that the impact velocity does not depend on the droplet diameter, the incoming liquid mass flux $\Phi_{i n c}$ is given by:

$$
\Phi_{\text {inc }}=\rho_{l} \Sigma d_{0} v_{i m p} / 2
$$

Combining equations 3 and 4 , and taking $d_{0}$ from PDPA measurements ${ }^{14}$, we can compute $v_{i m p}$ from $W_{c o r}$. For both probes, we find that the impact velocity is of order of several tens of centimers per second, about one order of magnitude smaller than the fluctuation $\delta v_{\text {r.m.s. }}$ of the axial velocity. Furthermore, the impact velocity tends to decrease as the axial velocity (and its fluctuation) increase. We have estimated the inertia effect discussed in $\S 3$, which is too small to correct $v_{i m p}$ by one order of magnitude. On the other hand, in a similar way to ref.[36], the reduction of $v_{i m p}$ could result from an interaction between droplets. Since the droplets concentration increases with the vapor velocity, this could also explain the lack of proportionality between the impact and the axial velocities. Alternatively, the impact velocity could be comparable to the vapor average transverse velocity, if the spray efficiency is less than unity. In this case, the bouncing or splashing probability would have to increase with velocity in order to account for our data. Such a dependence is qualitatively consistent with experimental studies of classical liquids spray droplets [12].

\footnotetext{
${ }^{14}$ These measurements are taken on the pipe axis. Several measurements at the height of the probes show that the diameters there do not differ from the on-axis value by more than $\pm 20 \%$.
} 


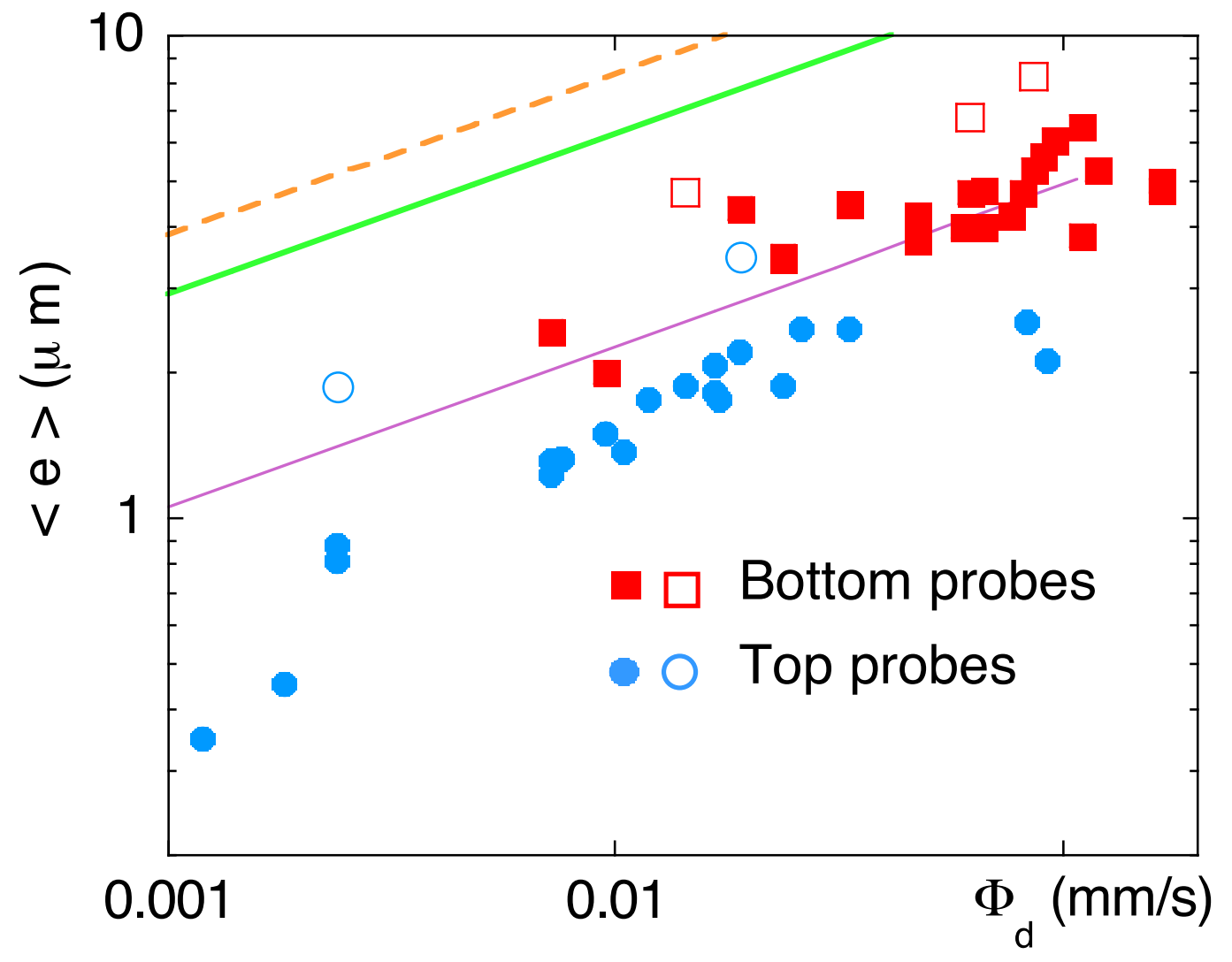

Figure 13: Thickness of the film deposited on the capacitive probes 10 and 5 as a function of the incident droplet flux, determined using the thermal probes located at the same height and eq. 3. $\mathbf{\square}$ : Bottom thermal probe ; •: Top thermal probe. Open symbols refer to normal fluid. The line through the data points correspond to a $1 / 3$ power law. The upper lines correspond to the predicted behaviour for normal helium, with a viscosity equal to the helium shear viscosity at $2.4 \mathrm{~K}$ (dashed line) and $1.8 \mathrm{~K}$ (continuous line).

The efficiency of deposition can also be probed through the thickness of the film deposited on the suspended capacitor. Indeed, this thickness is determined by the balance between the deposited flux $\Phi_{d}$ and the evacuated flux due to gravitational flow of the liquid along the wall and reatomization by the vapor flow. Assuming the normal and superfluid components to be locked, the vertical velocity $v(x, z)$ of the flowing film at distances $x$ to the probe plane and $z$ to the top of the probe, obeys the Navier-Stokes equation: 


$$
\eta\left(\frac{\partial^{2} v}{\partial z^{2}}+\frac{\partial^{2} v}{\partial x^{2}}\right)=\rho_{L} g
$$

where $\eta$ is the viscosity of the liquid, $\rho_{L}$ the liquid density, and $g$ the gravitational acceleration. Except very close to $z=0$, the $x$-derivative dominates and the velocity profile is parabolic in $x$. For each $z$, this relates the local average vertical velocity of liquid to the film thickness $e(z)$ through:

$$
<v>(z)=\frac{g}{6 \nu_{L}}(e(z))^{2}
$$

where $\nu_{L}$ is the liquid kinematic viscosity.

If reatomization can be neglected, the vertical flow at position $z$ equals the total deposited flux between $z=0$ and $z$, that is $z \Phi_{d}$ (neglecting the $z$ dependence of $\Phi_{d}$ over the probe height). This relates the thickness $e(z)$ to the deposited flux:

$$
e(z)=\left(\frac{6 \nu_{L}}{g} \Phi_{d} z\right)^{1 / 3}
$$

The thickness measured by the capacitive probe is obtained by averaging $e(z)$ over the active height of the probe $(6 \mathrm{~mm}$ high capacitive surface between $z=2 \mathrm{~mm}$ and $z=8 \mathrm{~mm}$ ). Like $e(z)$, it varies as the cubic root of the deposited flux ${ }^{15}$.

If the deposition efficiency is the same on heated and unheated walls, the thickness of the film deposited on the suspended capacitive probe should vary as the cubic power of the flux deposited on the top thermal probe ${ }^{16}$, as measured from its corrected CHF $W_{\text {cor }}$ (eq. 3). Figure 13 shows that this is approximately the case for thicknesses larger than $1 \mu \mathrm{m}$. Figure 13 also includes our data for the bottom thermal probe. In this case, there is no corresponding suspended capacitive probe, and we use instead the thickness measured by the capacitive probe (5), which is located on the pipe wall at the same height. Considering the transverse structure of the mist, this might lead to a factor of two error in the deposited flux. Within this factor of two, the data sets for the two thermal probes are consistent, extending the validity range of the power law behaviour to more than one decade in deposited flux.

\footnotetext{
${ }^{15}$ It also varies with the cubic root of the viscosity, which explains that the deposited film is much thinner, for similar fluxes, for helium than for water [37].

${ }^{16}$ The two probes, having a similar position within the pipe cross-section, are exposed to the same spray conditions.
} 
The validity of the power law behaviour, as well as the modest difference between the film thickness in the superfluid and normal states, confirms our hypothesis of a locked flow of normal and superfluid components. In other words, in contrast to the classical beaker flow experiment, the superfluid component does not seem able to flow separately from the normal one on the surface. Such a separation might be hindered by the gradient of chemical potential that it would probably induce in our geometry.

Quantitatively, the observed thickness is about three to four times less than predicted using reported values for the shear viscosity of helium [38]. Although this discrepancy could be explained by an error in our calculated conversion from capacitance to thickness ${ }^{17}$, such a level of error seems unlikely. A first interpretation of the discrepancy would be that it results from different deposited fluxes on both probes, due to a smaller efficiency of the deposition process on an unheated surface, as compared to the heated thermal probe. This could be the case if the bouncing probability is larger on a liquid film than on the dried surface of the heated probe at CHF. Experiments on millimeter size droplets of classical liquids do not show such a trend [14]. Another possibility is that, on a heated surface, an otherwise bouncing droplet could be evaporated during the bouncing event. This does not seem realistic. First, due to the $1 / 3$ exponent, quantitative agreement with theory would need the deposited flux on the capacitive probe to be 50 times less than on the thermal probe, which is quite large. Second, on theoretical grounds, for the heating powers applied, vaporization cannot occur on the time scale of bouncing. Indeed, the time necessary to evaporate a droplet of diameter $d$ lying on the surface scales with $d$ as $L \rho_{L} d / W_{s}$, where $W_{s}$ is the heating power per unit area at $\mathrm{CHF}$, set by the temperature difference between the metal plate and the saturated liquid, and the Kapitza resistance. Since, according to figure $9, W$ is of order several $\mathrm{mW} / \mathrm{cm}^{2}$, this time is of order $1 \mathrm{~s}$ for a droplet $1 \mu \mathrm{m}$ in diameter. This is orders of magnitude longer than the duration of the bouncing event $d / v_{i m p}$ for a droplet impinging the surface at a speed $v_{i m p}$, since $v_{i m p}$ is at least several $\mathrm{cm} / \mathrm{s}$.

The discrepancy could also arise if the deposited film is mainly evacuated by reatomization rather than by gravitational flow. In this case, however, one would expect reatomization to increase with the film thickness, resulting

\footnotetext{
${ }^{17} \mathrm{e} . \mathrm{g}$. due to an incorrect estimate of the thickness of glue between the wall and the kapton, or of the glue dielectric constant [30].
} 
in a thickness dependence on flux weaker than a cubic power, which we do not observe. A last possibility is that, for a given average thickness, the film drains faster than predicted by eq.6. Due to the non linear dependance of the velocity on the thickness, this is possible if the film is not uniform in the horizontal direction, which might happen if the deposited droplets fall faster than they spread. If this is the case, the lack of difference between the superfluid and normal data indicates that this is equally true in both cases.

To summarize, the CHF measured by the thermal probe is about one order of magnitude smaller than expected for a perfect spray efficiency of 1 and the assumption of an impact velocity of order the fluctuation of the vapor axial velocity. A dedicated spray cooling experiment with a controlled incident mass flux would be needed in order to directly measure the deposition efficiency, and, indirectly, to determine the impact velocity in the present case of turbulent spray deposition. On the other hand, the film thickness measured by the capacitive probe is smaller than expected for the deposited flux deduced from the CHF of the thermal probe, coupled with a simple lubrication model for a homogeneous, one-component, viscous film. Possible explanations are that the flux deposited on a wet, cold, surface is smaller than expected from the CHF on a dry surface, or that the film is not homogeneous, due to incomplete spreading of incident droplets. Settling between the two hypotheses is challenging, as it probably requires direct imaging of the impingement process and/or the film morphology, in both cases with a resolution of several micrometers. At any rate, our results show that neither the cooling capabilities of a given flux of droplets impinging a vertical surface, nor the draining of this surface, do strongly depend on the superfluid nature of the incoming liquid. If superfluidity modifies the spreading of droplets on the surface, it is not by a sufficient amount to affect these properties.

\subsection{Contribution of the mist to the global heat transfer}

The purpose of this section is to show that spray cooling explains the behaviour of the global heat transport through the pipe walls. On this aim, we use an approximate model to evaluate the temperature of the Kapitza box as a fonction of the applied power $W_{K}$, starting from the cooling power of the mist, measured using the suspended thermal probes.

In the superfluid phase, the deposited film is isothermal and at the temperature of the bulk liquid, so that the vaporization rate is uniform along the film and the liquid free surface. The film then extends up to a height $z_{0}$ such that the liquid deposition rate just equals this vaporization rate. Because 
the mist is stratified, $z_{0}$, hence the wetted surface, decreases with the applied heating power $W_{K}$, increasing the thermal resistance between the pipe walls and the diphasic flow. The temperature difference $\delta T\left(W_{K}\right)$ between the Kapitza box and the superfluid liquid in the pipe is given by:

$$
\delta T\left(W_{K}\right)=R_{K} W_{1} / S\left(z_{0}\right)
$$

where $W_{1}$ is the heat flux through the liquid (bulk or film), $R_{K}$ is the specific resistance between the box and the pipe, and $S\left(z_{0}\right)$ the wetted surface up to the height $z_{0}$. The heat flux per wetted unit area is uniform and equals $W_{1} / S\left(z_{0}\right)$, and the vaporization rate equals this flux divided by the latent heat. $z_{0}$ is set by the requirement that:

$$
W_{1} / S\left(z_{0}\right)=L \Phi_{d}\left(z_{0}\right)
$$

with $\Phi_{d}\left(z_{0}\right)$ the deposited flux per unit wall surface. Finally, $W_{K}$ is the sum of $W_{1}$ and the power needed to evaporate the droplets above $z_{0}$ :

$$
W_{K}=W_{1}+L \int_{z_{0}}^{\text {pipe top }} \Phi_{d}(z) d l(z)
$$

where $d l(z)$ is the length between $z$ and $z+d z$ along the wall cross-section. By scanning $z_{0}$ from the top of the tube down to the bulk liquid level, one computes $W_{K}$ from eqs.9 and 10 , and $\delta T\left(W_{K}\right)$ from eq.8, provided $\Phi_{d}(z)$ is known. As a simple evaluation, we take an exponential dependence for $\Phi_{d}(z)$, with the same characteristic height than measured for the interfacial density $\Sigma$ using the vertical laser beam $(\S 4.2)$. This assumes that the deposition velocity and the droplets size depend less on $z$ than $\Sigma$ does, and that the deposition velocity does not depend on the orientation of the wall surface. It also neglects the transverse structure of the mist. The prefactor of $\Phi_{d}(z)$ is set by the measured $\mathrm{CHF}$ of the bottom thermal probe, once corrected for the superflow effect.

We have carried out this procedure for a series of experiments at constant liquid level and temperature, and increasing vapor velocities. For these experiments, we have checked that the predicted $\Phi_{d}(z)$ at the height of the top thermal probe is in agreement with that directly measured using this probe, showing that the assumption of an exponential dependence is reasonable. Figure 14 compares the predicted and the measured behaviours for the different experimental conditions. For the calculated curves, we take a liquid 

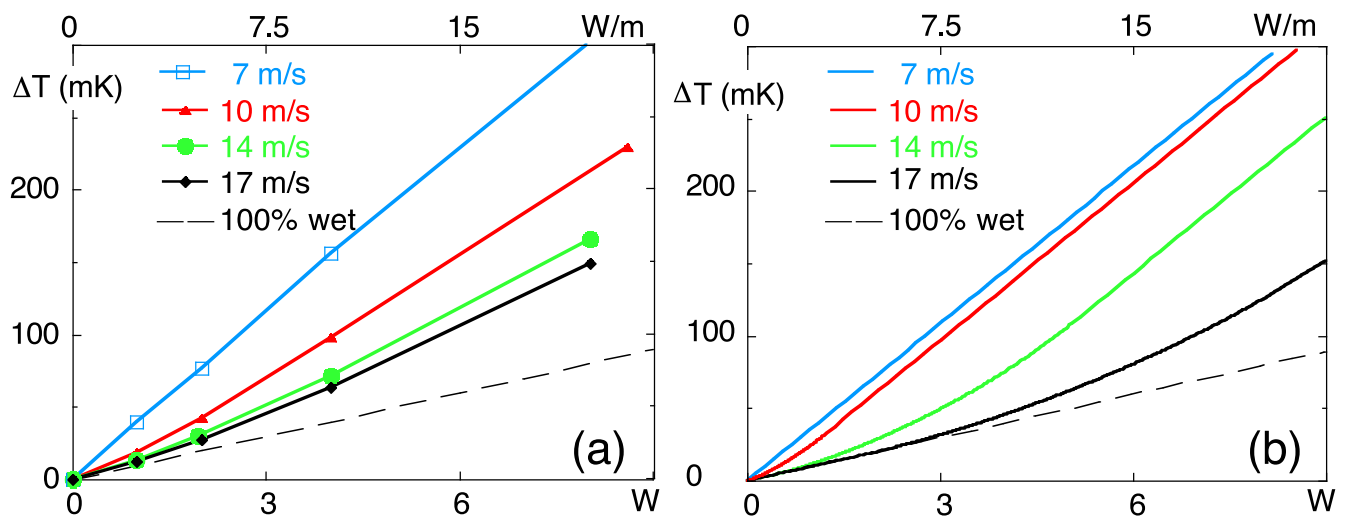

Figure 14: Global heat transfer through the pipe wall to the diphasic flow for vapor velocities ranging from $7 \mathrm{~m} / \mathrm{s}$ to $17 \mathrm{~m} / \mathrm{s}$, for an average bulk liquid level of $3.5 \mathrm{~mm}$ (corresponding to a wetted fraction equal to $20 \%$ of the total perimeter) and a temperature $T=1.8 \mathrm{~K}$. Comparison between the measured (a) and the calculated (b) heat transfer, based on the cooling power of the droplet mist measured by the suspended probes, and the theoretical model described in the text. The dashed line in both graphs corresponds to the fully wetted pipe. For the calculated curves, we take a liquid level of $7 \mathrm{~mm}$ so as to reproduce the measured exchange at $7 \mathrm{~m} / \mathrm{s}$ (see text). The model qualitatively accounts for the evolution from a linear to a non linear behaviour as the vapor velocity, hence the atomization, increases.

level of $7 \mathrm{~mm}$ (wetted fraction of $27 \%$ ) so as to reproduce the measured exchange at small velocity (weak atomisation). This height is larger than the $3.5 \mathrm{~mm}$ measured using the capacitive bottom probe (3). This difference can be due to the presence of a film deposited by surface waves above the average bulk level. If its thickness is in a range 10-50 $\mu \mathrm{m}$, it is thin enough to be only partly detected by the capacitive bottom probe (3) and thick enough to survive up to highest used heat fluxes ${ }^{18}\left(\simeq 100 \mathrm{~mW} / \mathrm{cm}^{2}\right.$ for $\left.7 \mathrm{~m} / \mathrm{s}\right)$.

For large axial velocities, the model accounts for the observation of a fully wetted behaviour at small heat fluxes, and for the non linearity at larger fluxes. It thus properly captures the role of the droplets mist in the thermal exchange. However, there are quantitative differences. First, the experimental global exchange improves faster than expected. Second, it sat-

\footnotetext{
${ }^{18}$ According to figure 13 , a film thickness of $10 \mu \mathrm{m}$ corresponds to a deposited flow rate equivalent to $500 \mathrm{~mW} / \mathrm{cm}^{2}$.
} 
urates earlier: it hardly changes between 14 and $17 \mathrm{~m} / \mathrm{s}$, whereas the cooling power measured by the thermal probes increases by $30 \%$ (resp. 100\%) for the bottom (resp. top) probe in the same velocity range. These two discrepancies with the observed behaviour likely stem from a difference in deposition rate between the walls and the suspended probes (positive at $10 \mathrm{~m} / \mathrm{s}$, negative at $17 \mathrm{~m} / \mathrm{s}$ ). The transverse dependence of the interfacial density alone cannot explain such a difference. Although optical measurements do show that the interfacial density is smaller, at a given height, close to the walls than to the suspended probes, the depletion is comparable for 10,14 and $17 \mathrm{~m} / \mathrm{s}$.

A saturation of the local thermal exchange, as measured by the bottom thermal probe, has also been seen at the largest velocity reached $(20 \mathrm{~m} / \mathrm{s})$. The critical heat flux in this case was the same than at $17 \mathrm{~m} / \mathrm{s}$, whereas the interfacial density facing the probe was nearly $50 \%$ larger. This suggests that the mechanism limiting the heat exchange with walls has to do with either the axial velocity (reatomization of the film) or a decrease of the deposition rate, again possibly due to interactions between droplets at large densities [36]. It is however not clear why these processes would affect the pipe walls earlier than the probes located in the pipe central region.

This shows that, while the non linearity of the global heat transport is qualitatively understood, it cannot be precisely predicted from local measurements in the central region of the pipe only, due to the complexity of the deposition mechanism.

\subsection{Possible application of spray cooling to an LHC upgrade}

In the case of LHC, the thermal exchange should warrant that the magnets operate at their nominal temperature of $1.9 \mathrm{~K}$. This constraint is a major issue for the final focus magnets (inner triplets) which focalise the beams in the interaction region. In the present version of LHC, the linear heat load on these magnets due to beam-induced heating is up to $10 \mathrm{~W} / \mathrm{m}$, an order of magnitude larger than for the arcs magnets, over a distance of $30 \mathrm{~m}$ [39]. The corresponding integrated heat load is nearly $200 \mathrm{~W}$. While this is comparable to the heat load used in our experiments, the vapor velocity is much smaller due to a larger pipe diameter $(\simeq 90 \mathrm{~mm})$ so that atomization probably does not take place here. For these parameters, experiments on a full-scale prototype [40, 41] have shown that, in the stratified regime, a total mass flow of $13 \mathrm{~g} / \mathrm{s}$ provides a wetted area of about $20 \%$, sufficient for meeting the required thermal exchange (temperature rise of order $50 \mathrm{mK}$ for $10 \mathrm{~W} / \mathrm{m}$ ). However, this will not stay true in an upgraded version of LHC 
with an up to ten fold increase of luminosity. In this case, the linear losses are expected to be in the range $20-100 \mathrm{~W} / \mathrm{m}$, depending on the upgrade level [5]. As a consequence, if the pipe diameter is not increased for the LHC upgrade, the integrated losses, hence the vapor velocity will increase, and atomisation could set in. Two questions then arise. First, can the increase in the wetted surface due to atomisation stand the expected linear losses? Second, how does the droplets mist increase the longitudinal pressure drop, hence the upstream temperature of the saturated liquid? Our study gives some hints. Concerning the heat exchange, an extrapolation of figure 14 suggests that, at $1.8 \mathrm{~K}$ the temperature increase due to a linear heat load of $30 \mathrm{~W} / \mathrm{m}\left(W_{K}=12 \mathrm{~W}\right)$ is less than $0.25 \mathrm{~K}$ in the strongly atomized regime. This was confirmed by one direct measurement at a vapor velocity of $14 \mathrm{~m} / \mathrm{s}$. In such conditions, the magnet bath temperature remains superfluid. This would allow proper operation if the final focus magnets could work at, e.g, $2.1 \mathrm{~K}$, rather than $1.9 \mathrm{~K}$. As for the pressure drop, it was measured between the two pipe ends, over a distance of about $10 \mathrm{~m}$. For all experimental conditions, it scales as the vapor kinetic energy, with a slope twice that expected for monophasic turbulent flow [9, 29]. Analysis of the data suggests that the excess dissipation is mainly due to the free surface between the liquid and the vapor, rather than to the droplets acceleration by the vapor. In any event, the maximal pressure drop for a vapor velocity of nearly $20 \mathrm{~m} / \mathrm{s}$ is about $400 \mathrm{~Pa}$ over $10 \mathrm{~m}$, corresponding to a temperature increase of $150 \mathrm{mK}$ over the full length of the inner triplets $(30 \mathrm{~m})$. This increase is too large, but should be strongly reduced in the LHC case due to the much larger pipe diameter.

This larger pipe diameter can also be expected to increase the surface wetted by the film with respect to our experiment. This gives reasonable hope that the present design could accommodate a significant increase of the linear heat load. However, the preceding section also shows that the efficiency of spray cooling is difficult to predict precisely. Direct measurements of the global heat transport on a full-scale prototype are necessary to check the applicability of such a solution.

\section{Conclusions}

We have presented a quantitative study of the contribution of spray cooling to heat transfer between an atomized diphasic flow and the pipe walls. Our results show that the critical heat flux (CHF) for a heated surface, as 
well as the thickness of the film deposited on an unheated vertical surface, increase with the spray interfacial density. The CHF is however smaller than expected, showing that, either the impact velocity of droplets on walls is smaller than the transverse vapor velocity, or that these droplets have a large probability to bounce on the dried surface walls. The deposited film is also thinner than expected, either because the bouncing probability is larger on a wet surface than on a dry one, or because the film thickness is non uniform, resulting in a faster draining. In any case, superfluid droplets are not found more efficient than normal droplets for the purpose of spray cooling. For given mist conditions in terms of droplets size and velocity, the CHF, once corrected for the effect of lateral superflow, and the film thickness, is the same in the normal and the superfluid phases. This suggests that the spreading or rebound dynamics of superfluid droplets do not significantly differ from those of normal droplets, at least as far as they influence the above properties. This intriguing conclusion would deserve to be checked using a dedicated experiment, where the droplet flux would be directly controlled rather than being the result of a complex atomization phenomenon. The interest of the present experiment, however, is that it is representative of the large-scale cooling system of LHC. From this point of view, our study shows that spray cooling does improve the thermal exchange with walls due to the formation of a liquid layer, but that this improvement is fragile, due to the stratification of the mist. Furthermore, because of the complex nature of the diphasic flow, this improvement cannot be precisely predicted from local measurements of the critical heat flux only. Real size measurements of global thermal exchange seem mandatory to evaluate whether spray cooling can be used to evacuate the large linear heat loads expected in the context of an upgrade of the LHC, as well as to check that, in this case, the longitudinal pressure drop induced by the mist production remains bearable.

\section{Acknowledgments}

We are indebted to M. Bon Mardion and the $400 \mathrm{~W}$ team for their crucial help. We thank E. Di Muoio for her participation to the early experiments, R. van Weelderen and P. Lebrun for many stimulating discussions, and CERN for financial support.

[1] Kim, J.. Spray cooling heat transfer: The state of the art. Int J Multiphase Flow 2007;28:753-767. 
[2] Hedayat, A., Hastings, L., Bryant, C., Plachta, D.. Large scale demonstration of liquid hydrogen storage with zero boiloff. Adv Cryog Eng (AIP Conf Proc 613) 2002;47B:1276-1283.

[3] Lebrun, P., Serio, L., Tavian, L., Van Weelderen, R.. Cooling strings of superconducting devices below $2 \mathrm{k}$ : the helium II bayonet heat exchanger. Adv Cryog Eng 1998;43A:419-426.

[4] Lebrun, P.. Cryogenics for the large hadron collider. IEEE Transactions on Applied Superconductivity 2000;10:1500-1506.

[5] Brüning, O.S., Cappi, R., Garoby, R., Gröbner, O., Herr, W., Linnecar, T.P.R., et al. Lhc luminosity and energy upgrade: A feasibility study. oai:cds.cern.ch:601847. Tech. Rep. LHC-Project-Report-626. CERN-LHC-Project-Report-626; CERN; Geneva; 2002.

[6] Rousset, B., Grimaud, L., Gauthier, A., van Weelderen, R.. He II two-phase flow in an inclinable $22 \mathrm{~m}$ long line. Adv Cryog Eng 2000;45B:1009-1016.

[7] Di Muoio, E., Jager, B., Puech, L., Rousset, B., Thibault, P., van Weelderen, R., et al. Optical investigations of He II two-phase flow. Adv Cryog Eng (AIP Conf Proc 613) 2002;47B:1675-1682.

[8] Rousset, B., Jager, B., Di Muoio, E., Puech, L., Thibault, P., Vallcorba, R., et al. He II co-current two phase flow at high vapor velocities. Adv Cryog Eng (AIP Conf Proc 613) 2002;47B:1311-1318.

[9] Rousset, B., Thibault, P., Perraud, S., Puech, L., Wolf, P.E., van Weelderen, R.. Heat transfer enhancement of He II co-current two-phase flow in the presence of atomisation. Proceedings of the International Cryogenic Engineering Conference-ICEC-20 (Elsevier) 2005;:761-764.

[10] Marmottant, P., Villermaux, E.. On spray formation. J Fluid Mech 2004;498:73-111.

[11] Paras, S., Karabelas, A.. Droplet entrainement and deposition in horizontal annular flow. Int J Multiphase Flow 1991;17:455-468.

[12] Mundo, C., Sommerfeld, M., Tropea, C.. Droplet-wall collisions experimental studies of the deformation and breakup process. Int $\mathrm{J}$ Multiphase Flow 1995;21:151-173. 
[13] Karl, A., Frohn, A.. Experimental investigation of interaction processes between droplets and hot walls. Physics of fluids 2000;12:785-796.

[14] Cossali, G., Coghe, A., Marengo, M.. The impact of a single drop on a wetted solid surface. Experiments in fluids 1997;22:463-472.

[15] Moreira, A.L.N., Moita, A.S., Panao, M.R.. Advances and challenges in explaining fuel spray impingement: How much of single droplet impact research is useful? Progress in energy and combustion science 2010;36:554-580.

[16] Reeks, M.W.. Dispersion of small particles suspended in an isotropic turbulent fluid. J Fluid Mech 1977;83:529-546.

[17] Pan, L., Hanratty, T.. Correlation of entrainment and deposition in horizontal annular flow. Int J Multiphase Flow 2002;28:385-408.

[18] McCoy, D., Hanratty, T.. Rate of deposition of droplets in annular two-phase flow. Int J Multiphase Flow 1975;3:319-31.

[19] BenRayana, F.. Contribution à l'étude des instabilités interfaciales liquide-gaz en atomisation assistée et tailles de gouttes. Ph.D. thesis; Institut National Polytechnique de Grenoble; 2007.

[20] Simmons, M.J.H., Hanratty, T.J.. Droplet size measurements in horizontal annular gas-liquid flow. Int J Multiphase Flow 2001;27:861-883.

[21] Joanny, J.F.. Spreading of superfluid drops. J Phys (Paris) 1985;46:807813.

[22] Roussel, P., Girard, A., Jager, B., Rousset, B., Bonnay, P.. The $400 \mathrm{~W}$ at $1.8 \mathrm{~K}$ test facility at cea-grenoble. Adv Cryog Eng (AIP Conf Proc 823) 2006;51:1420.

[23] Thibault, P., Perraud, S., Rousset, B., Puech, L., van Weelderen, R., Wolf, P.E.. A new experiment to explore He II two-phase flow behavior at high mass flow rate. Proceedings of the International Cryogenic Engineering Conference-ICEC-19 (Narosa publishing house, New Dehli, India), 2002;:825-828. 
[24] Perraud, S., Rousset, B., Thibault, P., van Weelderen, R., Wolf, P.E.. Comparison between normal and He II two-phase flows at high vapor velocities. Adv Cryog Eng (AIP Conf Proc 823) 2006;51B:1669-1676.

[25] Wolf, P.E., Bonnet, F., Guyon, L., Lambert, T., Perraud, S., Puech, L., et al. Probing helium interfaces with light scattering: From fluid mechanics to statistical physics. Eur J Phys E 2009;28:183-198.

[26] Wolf, P., Di Muoio, E., Thibault, P., Puech, L., Rousset, B., van Weelderen, R.. Pdpa characterization of the droplet mist in a He II two-phase flow. Int Cryo Eng Conf 2003;:667.

[27] Thibault, P., Di Muoio, E., Perraud, S., Rousset, B., Puech, L., van Weelderen, R., et al. Mist observation in superfluid He two-phase flow by pda techniques. 2004, URL http://Itces.dem.ist.utl.pt/lxlaser/lxlaser2004/pdf/paper_06_4.pdf.

[28] Rousset, B., Chatain, D., Puech, L., Thibault, P., Viargues, F., Wolf, P.E.. Visualization in cryogenic environment: Application to two-phase studies. Cryogenics 2009;49:554-564.

[29] Perraud, S.. Thermo-hydraulique d'un écoulement horizontal d'hélium superfluide diphasique. Ph.D. thesis; Université Joseph-Fourier, Grenoble; 2007.

[30] Thibault, P., Diribarne, P., Fournier, T., Puech, L., Perraud, S., Puech, L., et al. On the design of capacitive sensors using flexible electrodes for multipurpose measurements. Rev of Scient Instr 2007;78:043903.1-043903.7.

[31] Thibault, P., Di Muoio, E., Puech, L., Rousset, B., Wolf, P.. Probing the wetted perimeter in a He II two-phase pipe-flow experiment using a capacitive sensor. Adv Cryog Eng (AIP Conf Proc 613) 2002;47:16831682.

[32] Rousset, B., Di Muoio, E., Thibault, P., Puech, L., van Weelderen, R., Wolf, P.. Thermohydraulic behaviour of He II in stratified co-current two-phase flow at high vapor velocities. Proceedings of the International Cryogenic Engeneering Conference-ICEC-19 (Narosa publishing house, New Dehli, India, 2003) 2003;:805-808. 
[33] Turkington, R.R., Edwards, M.H.. Discrete flow rates of the helium film. Phys Rev 1968;168:160-162.

[34] Turkington, R.R., Harris-Lowe, R.F.. Reproducible flow-rate structure in He-4 films. Physica B 1990;165(Part 1):587-588.

[35] Harris-Lowe, R.F., Turkington, R.R.. Distribution of He-4 film flowrates. Physica B 1994;194(Part 1):645-646.

[36] Hay, K., Liu, Z., Hanratty, T.. Relation of deposition to drop size when the rate law is non-linear. Int J Multiphase Flow 1996;22-5:829848.

[37] Williams, L., Dykhno, L., Hanratty, T.. Droplet flux distributions and entrainment in horizontal gas-liquid flows. Int J Multiphase flow 1996;22:1-18.

[38] Wang, S., Howald, C., Meyer, H.. Shear viscosity of liquid-he4 and he3-he4 mixtures, especially near the superfluid transition. J Low Temp Phys 1990;79:151-187.

[39] Byrns, R., Huang, Y., Kerby, J., Lebrun, P., Morrison, L., Nicol, T., et al. LHC Project Report 219 - The Cryogenics of the LHC Interaction Region Final Focus Superconducting Magnets. CERN; 1998.

[40] Darve, C., Huang, Y., Nicol, T., Peterson, T.. Experimental investigations of He II heat transfer through a short section of LHC inner triplet quadrupole heat exchanger. IEEE Transactions on Applied Superconductivity 2001;11:1629 - 1632 .

[41] Darve, C., Blanco, E., Huang, Y., Nicol, T., Peterson, T., van Weelderen, R.. He II heat exchanger test unit for the LHC inner triplet. In: AIP Conference Proceedings. 613A; Fermi Nat. Accel. Lab., Batavia, IL, USA; 2002, p. $147-154$. 